\title{
Automated Energy Distribution and Reliability System (AEDR): Final Report
}

Subcontract Report NREL/SR-581-43674 July 2008
D.L. Buche

Northern Indiana Public Service Company Merrillville, Indiana

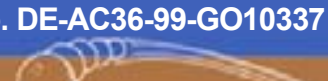




\section{Automated Energy Distribution and Reliability System (AEDR): Final Report}

D.L. Buche

Northern Indiana Public Service Company

Merrillville, Indiana

NREL Technical Monitor: T. Basso

Prepared under Subcontract No. RAX-4-33651-01

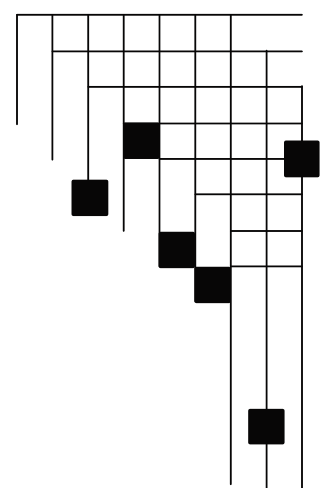


This publication was reproduced from the best available copy submitted by the subcontractor and received no editorial review at NREL

\section{NOTICE}

This report was prepared as an account of work sponsored by an agency of the United States government. Neither the United States government nor any agency thereof, nor any of their employees, makes any warranty, express or implied, or assumes any legal liability or responsibility for the accuracy, completeness, or usefulness of any information, apparatus, product, or process disclosed, or represents that its use would not infringe privately owned rights. Reference herein to any specific commercial product, process, or service by trade name, trademark, manufacturer, or otherwise does not necessarily constitute or imply its endorsement, recommendation, or favoring by the United States government or any agency thereof. The views and opinions of authors expressed herein do not necessarily state or reflect those of the United States government or any agency thereof.

Available electronically at http://www.osti.gov/bridge

Available for a processing fee to U.S. Department of Energy and its contractors, in paper, from:

U.S. Department of Energy

Office of Scientific and Technical Information

P.O. Box 62

Oak Ridge, TN 37831-0062

phone: 865.576.8401

fax: 865.576 .5728

email: mailto:reports@adonis.osti.gov

Available for sale to the public, in paper, from:

U.S. Department of Commerce

National Technical Information Service

5285 Port Royal Road

Springfield, VA 22161

phone: 800.553 .6847

fax: 703.605.6900

email: orders@ntis.fedworld.gov

online ordering: http://www.ntis.gov/ordering.htm 


\section{Preface}

In 1998, the Department of Energy (DOE) established the Distributed Power Program to address systems integration issues and market barriers that may prohibit the widespread deployment of distributed power technologies. Initial efforts under the program involved creating national technical interconnection standards, establishing research and development programs to address system integration technologies documenting regulatory and institutional market barriers, and working with industry and state and federal policymakers to remove barriers. At that time, the National Renewable Energy Laboratory (NREL) led these research activities for the DOE. Under this subcontract, Northern Indiana Public Service Company (NIPSCO), a NiSource, Inc. Company, has developed a modernized Automated Energy Distribution and Reliability System (AEDR) based on geographical information system (GIS) technology. This integrated geographical database serves to enhance energy supply reliability and security by improving the integrity and accessibility of location data, while fostering public safety through sharing of utility location information with authorized government entities and other organizations. This modernization of the gas \& electric infrastructure helps to assure safe, reliable, and affordable service to homes and businesses.

This Final Report is the culmination of the efforts of many technical and non-technical professionals who spent thousands of hours of careful planning and execution which led to the development of this state-of-the-art geographical information system, the AEDR. The AEDR manages both spatial and non-spatial NIPSCO data in a manner that enables safety by ensuring data integrity through validation and by providing easy accessibility to "all of the data, all of the time."

Other NREL reports provide more details: "Automated Energy Distribution and Reliability System Status Report” http:/www.nrel.gov/docs/fy08osti/42265.pdf and, "Automated Energy Distribution and Reliability System (AEDR): Validation Integration - Results of Future Architecture Implementation" NREL Report No. SR-581-43432, that includes the NIPSCO-NREL “Wind Generation Integration Study (June 2007).

NIPSCO wishes to express sincerest gratitude for the funding assistance provided by the Department of Energy and the excellent technical support received by the National Renewable Energy Laboratory. This project would not have been possible without their support.

NIPSCO also wishes to thank the NIPSCO GIS project team and their subcontractors; SSPInnovations, GreatArc Technologies, QC Data, Telvent Miner \& Miner, ESRI, Enspiria Solutions Inc., NIPSCO subject matter experts and all who participated in the development and implementation of the AEDR. 


\section{Table of Contents}

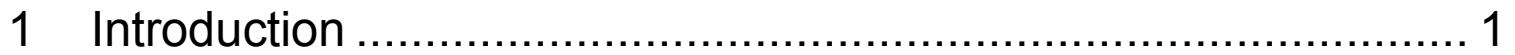

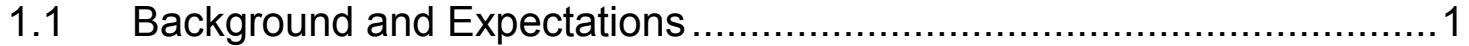

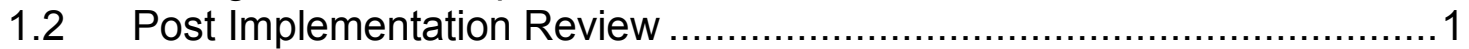

2 Final Report Summary ..................................................... 2

2.1 Technology Platform and System Environment..............................2

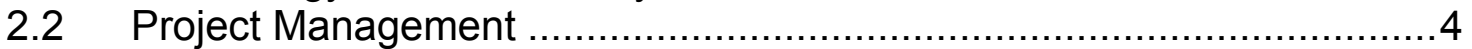

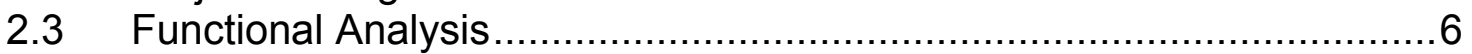

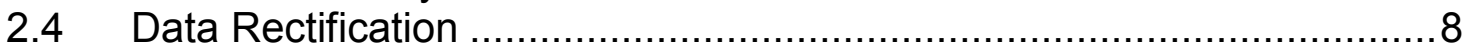

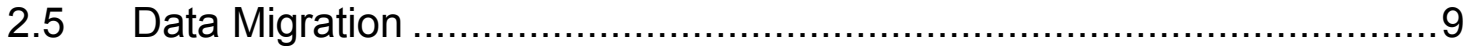

2.6 ArcFM / ESRI Configuration ..................................................... 11

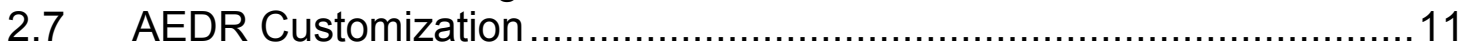

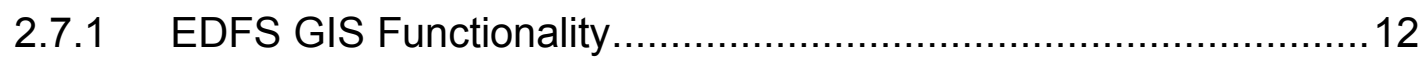

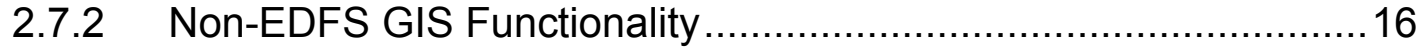

2.7.3 GIS to CAD Conversion.............................................................

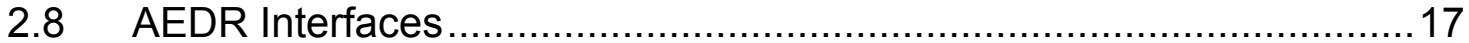

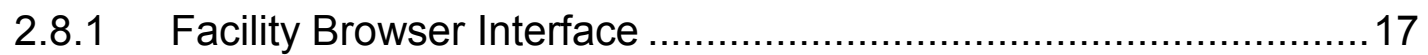

2.8.2 Field Browser Interface........................................................ 18

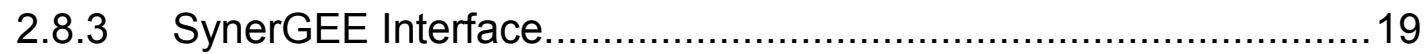

2.8.4 CADOPS and FeederALL Interfaces ....................................20

2.8.5 Material Accounts Payable and Purchasing System (MAPPS)

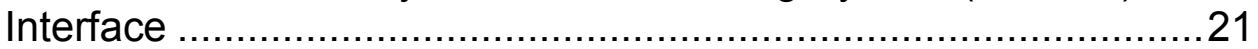

2.8.6 Customer Information System (CIS) Interface ...........................21

2.8.7 General Ledger Interface ................................................22

2.8.8 Engineering Accounts Information File (EAIF) Interface (Indirect). 22

2.8.9 Material and Labor Online Guide (MLOG) Interface .....................22

2.9 System Performance Evaluation / Stress Testing..............................23

2.10 AEDR Online Help Documentation ...............................................23

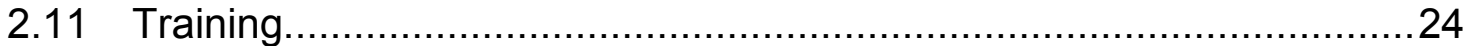

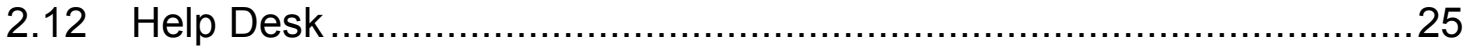

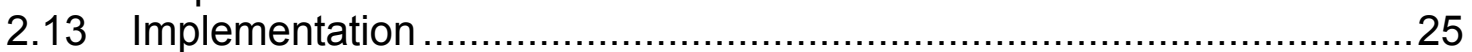

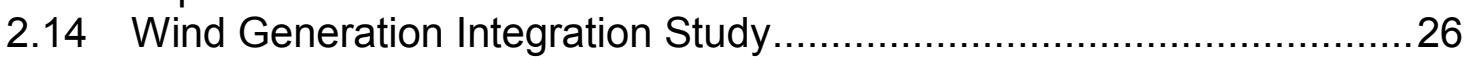

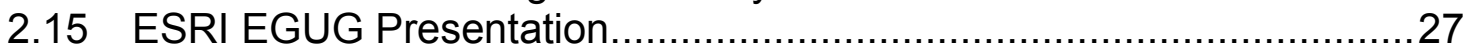

3 Conclusion .................................................................... 28 


\section{Introduction}

This final report summarizes the development and implementation of the Automated Energy Distribution and Reliability (AEDR) system. The details for all of the topics of this report, and additional templates, tools and ideas can be found in the following reports previously submitted to NREL:

- Validation Integration - Results of Immediate Architecture Implementation

- Validation Integration - Results of Future Architecture Implementation

- NIPSCO Wind Generation Integration Study

\subsection{Background and Expectations}

Traditionally residing partly in an AutoCAD-based system and partly in a relational database system, NIPSCO facility information (gas, electric and land) was served by distinct and not readily interoperable technologies. Thus it was time consuming and sometimes cumbersome to readily maintain the integrity of the data and disseminate facility information to those requiring it. "All of the data, all of the time" was not readily accessible by NIPSCO. The purpose of this project was to implement a database-driven GIS solution that would manage all of NIPSCO's gas, electric and landbase objects.

The goals of this project included:

- Elimination of redundant data and systems

- Implementation of a business rule-driven application to manage GIS data

- Improvement of data quality and integrity

- Gas and electric load study integration

- Establishment of improved and integrated information to satisfy external entities' needs and identify opportunities such as

1. Identification of critical habitats of threatened or endangered species

2. Exploration of alternative energies, such as wind energy, combined heat and power (CHP) units and distributed resources/generation (DR/DG)

3. Impact of future installation of facilities located near the Indiana Dunes National Lakeshore to enable environmentally sound decisions.

- Gather and provide information to, and support participation in IEEE standards development work groups.

\subsection{Post Implementation Review}

A post implementation review of the AEDR system implementation was conducted. Those findings are included in Appendix A. The post implementation review is an assessment of:

- how closely the stated objectives and deliverables were met

- the benefits derived, and

- the effectiveness of the solution and the lessons learned for future project improvements. 


\section{Final Report Summary}

\subsection{Technology Platform and System Environment}

After an extensive Request for Proposal (RFP) and vendor selection process, Environmental Systems Research Institute (ESRI) and Telvent Miner \& Miner were chosen to provide the GIS architecture for NIPSCO's AEDR.

ESRI's ArcGIS and Telvent Miner \& Miner's ArcFM technologies replaced many of NIPSCO's legacy systems. ArcMap with ArcView, ArcEditor/ArcFM and ArcInfo combinations for data viewing and maintenance and application development and maintenance, respectively are used to support the AEDR.

The two major server components include the database servers and the Citrix server farm. The database servers host the data stores and the ArcSDE server components that provide spatial access to the data stores. The Citrix servers are the application service providers.

Seven SQL Server instances split out over two database servers were utilized to create well-defined separation between the project initiatives. Six of which are:

- Delta (only contains the latest database schema, not data)

- Migration

- Development

- Test One

- Test Two

- Production

A second production server and the seventh instance were added when the Facility Browser came on-line.

This allowed the project team to manage database and application updates and testing in a methodical manner while allowing multiple major initiatives to be pursued in parallel.

Microsoft SQL Server is the underlying RDBMS. ESRI ArcSDE sits on top of the database and manages all GIS interaction with the database. We chose SQL Server over Oracle for reasons that SQL Server was an affordable and viable option, a decision that resulted in a large cost savings in both database software and continued support, as SQL Server was considerably easier to manage. The final AEDR solution uses two production SQL Servers and one test SQL Server, all running ArcSDE.

Database privileges for the AEDR require users to have permissions set on each database object. Therefore, system roles were created and users were assigned to the appropriate role. All components of the AEDR system use windows authentication so that the end user is never required to enter a password. Windows authentication allows the application to use the credentials of the users logged on to the computer where the application is being used. 
The AEDR project utilizes a production Citrix farm including four Windows 2003 servers and a single development / test Citrix server. Citrix MetaFrame, a Windows Terminal Server add-on, significantly enhances the functionality, manageability and ease of deployment of Terminal Server solutions. Windows Terminal Server is a multi-user server operating system with the capability of hosting multiple simultaneous thin-client sessions on remote client devices. All client processing is performed locally at the Terminal Server.

We have established three Windows Active Directory groups of Citrix users defined with varying access. The groups are used to set the access level for each of the published Citrix applications. This ensures that only authorized users can access each application with each defined permission level. The same applications are made available to more than one group but the permission level is changed depending on the authorization of the user. The permissions can be altered for both ESRI and Telvent Miner \& Miner applications by changing the license available to the user.

Licensing is automated via the NIPSCO custom ArcLauncher application. It is a command line application that accepts parameters for the application to be run (i.e. ArcMap, ArcCatalog, etc.) and the license level to be used. It then sets both the ESRI license environment variable and the Miner \& Miner registry keys appropriately before starting the application.

ArcIMS is used to deliver Facility Browser maps, data and services via the Web. A second database server running ArcSDE was required to ensure optimal performance. The production data is replicated from the main database server on a nightly basis.

Figure 2-1 represents the AEDR system environment. 


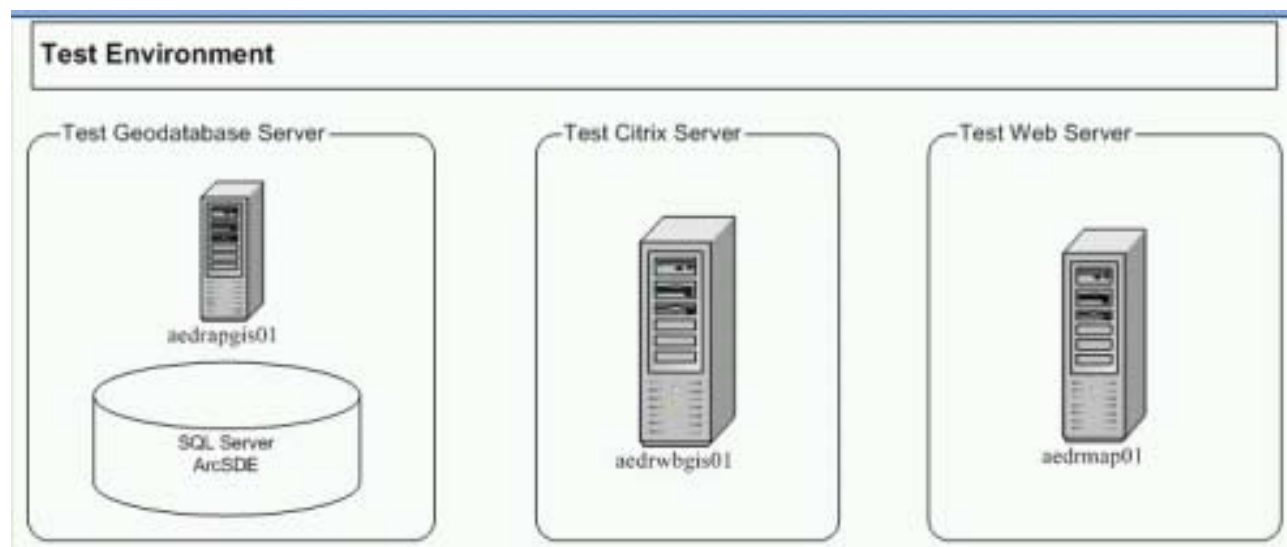

Production Environment
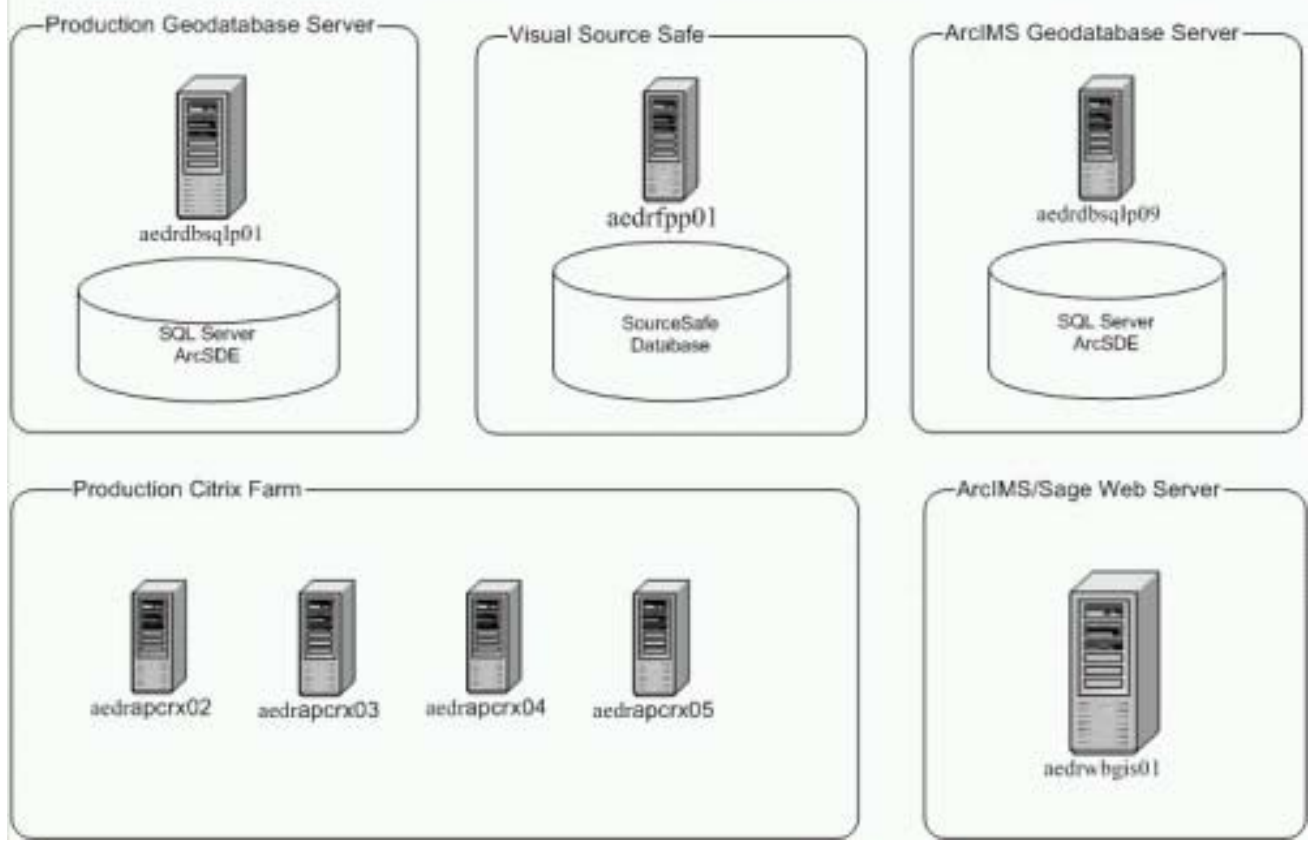

Figure 2-1. NIPSCO Server Environment

\subsection{Project Management}

The AEDR project was highly organized, had strength in project sponsorship and adherence to system development life cycle industry standards which helped to ensure its success. Some of the tasks that enabled the achievements of this project are: Project scope and critical path components were controlled, project budget and timeline were maintained, quality assurance was of prime focus, detailed test cases were thoroughly exercised for every application, risks were managed throughout the project and project decisions were made in a timely manner. Problems were tracked and resolved as timely as possible and resources were assigned team lead roles to ensure ownership. 
Project sponsors, a project manager, GIS developers, GIS analysts, GIS data migration vendors, IT database support, IT server support and subject matter experts in Maps \& Records, Engineering and individuals responsible for interfacing systems all participated in the development of various elements of the AEDR system.

The project was divided into components that were developed simultaneously and progressively. Some of these components had interdependencies, others did not. Each component was assigned a resource (team lead) responsible for ensuring the success of that component:

- ArcFM Configuration

- ArcFM Customization

- Data Migration and Rectification

- Data Modeling

- Development, Testing and Production Environments (administration)

- Documentation and Training

- Problem Tracking and Management

- Interface Development

- System Architecture

- Library Administration

- Testing

- Implementation

Well-developed and maintained Plans based on system development life cycle industry standards kept the project on track, the team engaged and ensured tasks did not slip through the cracks. Although other "Plans" existed, some of the more key Plans were essential to support the development of the AEDR are listed here:

- Inception Project Plan

- Elaboration Project Plan

- Construction Project Plan

- Deployment Project Plan

- Implementation Plan

- Migration Plan

- Risk Management Plan

- Change Control Plan

- Field Browser Implementation Plan

The project budget was established through the development of software, licensing, hardware, individual component and contractor and internal resource services estimates. Twenty percent contingency covered unexpected expenditures.

Both software and hardware analysis was conducted to determine software adequacy and hardware requirements. We requested (and were granted) trial licenses for a proof of concept activity before entering into a perpetual licensing arrangement with ESRI and Telvent Miner \& Miner. The results of the trial period provided sufficient proof of 
concept to continue the project. The hardware analysis provided the number of servers and server configuration required to support the development, testing and final implementation of the AEDR. Subsequently, performance testing verified the database and Citrix server configurations would support the AEDR in production.

\subsection{Functional Analysis}

Functional analysis began with a broad look at the project goals and then refinement of each area within the project with additional detail, functional requirements, software requirements, and finally a functional software design. As the process surrounding each area of the project became more detailed, it was tied back to the higher level components to ensure consistency, scope, and coverage.

The objectives of the AEDR functional analysis are shown in Figure 2-2.

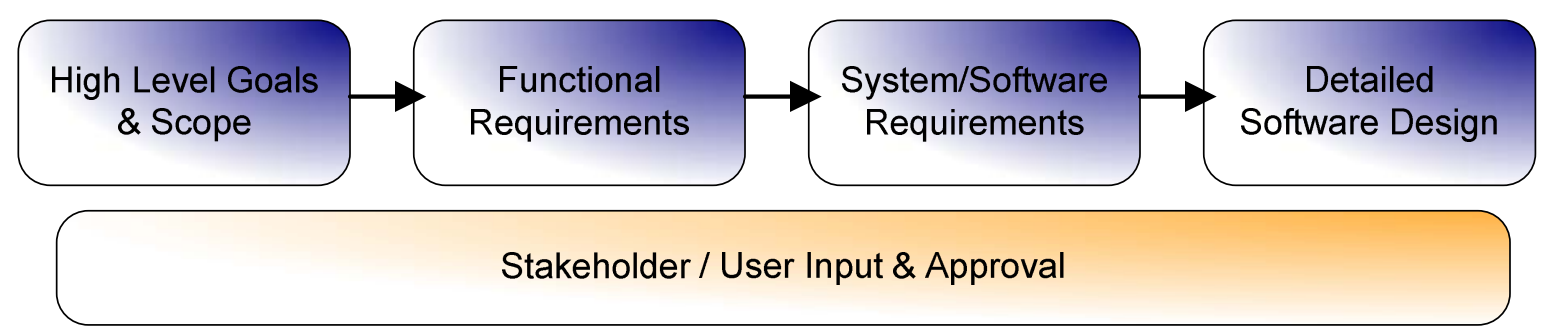

Figure 2-2. The functional analysis diagram

Extensive interviews were conducted with the identified stakeholders and / or users for all of the functional areas. From the interviews, use cases were developed to define the desired workflow and interaction in each of these areas. This then drove the creation of functional requirements which were organized and documented into spreadsheets. These requirements were entered into a product called System Architect which generated Figure $2-3$, the functional systems diagram. 


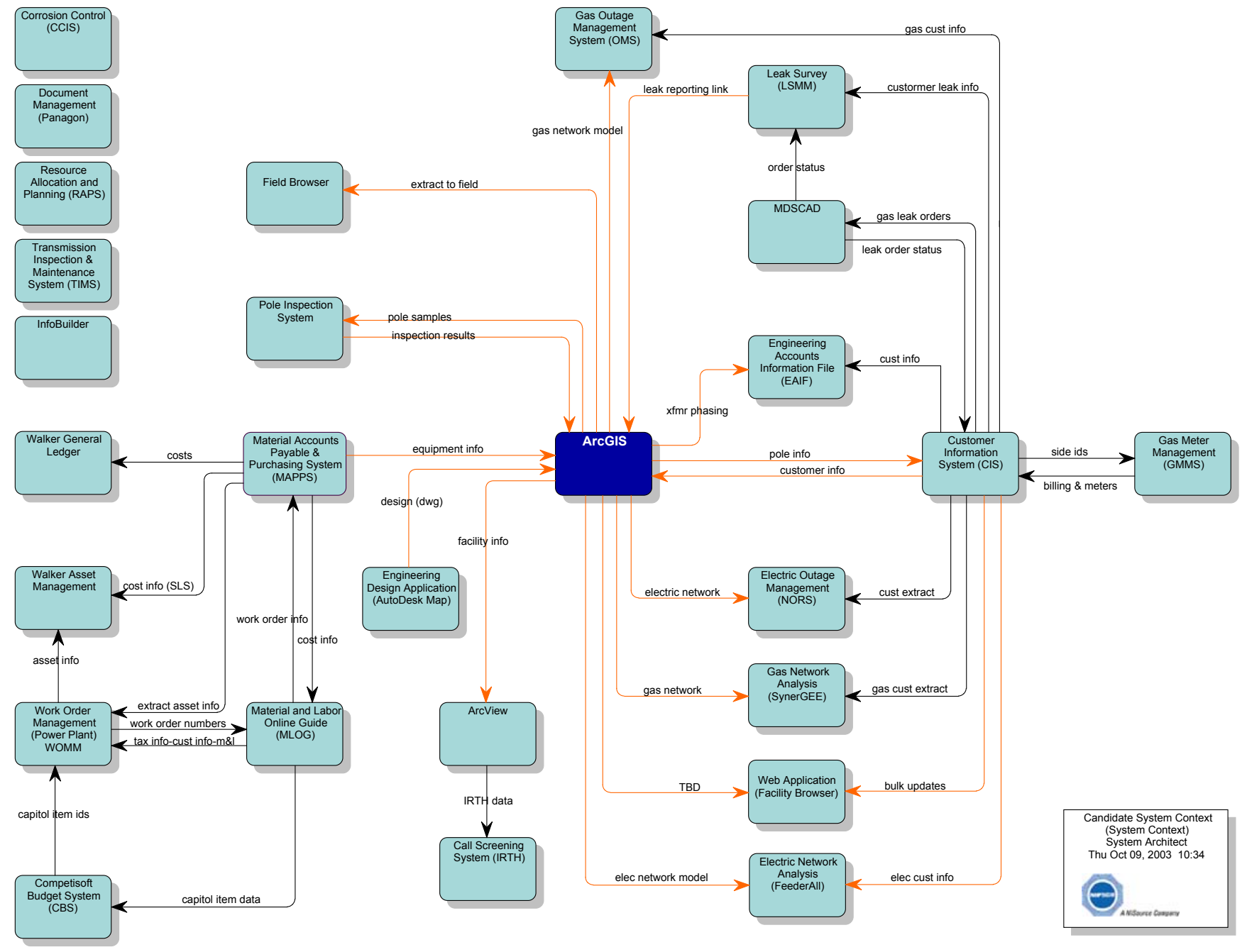

Figure 2-3. Functional systems diagram

There are several systems listed on this diagram that are not included within the functional areas of this project. These are included to completely identify the systems surrounding this project.

Software-specific concepts were developed with input from the stakeholders / users. These concepts provided the basis for the system requirements for each functional area which detailed how the system would operate, the intended user interaction, any custom development that was required, and which technological approaches would be used. 


\subsection{Data Rectification}

NIPSCO's objective in pursuing the data rectification project was to improve the positional accuracy of its AutoCAD data prior to the migration of the data into the new GIS.

The landbase data within NIPSCO's legacy AutoCAD files was converted from sources of moderate positional accuracy and variable currency. Some landbase and facility feature positions were essentially correct, many land and facility feature positions were modestly incorrect when compared to more accurate sources, and some were more dramatically misplaced.

The size of this effort was significant in that NIPSCO did not have the manpower to achieve this task within the scope of this project to complete the implementation within the required timeframe so the work was contracted externally.

Digital Orthophoto Quarter Quadrangle (DOQQ) data provided for consistent and comprehensive coverage of the NIPSCO service territory and was used as the positional control source.

The legacy source coordinate system and datum (Indiana State Plane West NAD27) was upgraded by re-projection to the Indiana State Plane West NAD83 coordinate system.

Rectification preparation began in June 2004. Throughout the next six months, rectification rules were refined, automated quality assurance processes were developed and tested, quality assurance procedures were established, the NIPSCO quality assurance team was trained, ancillary issues were identified, procedures were established to manage the rectification process and the data was reprojected to the new coordinate system. Modifications to the preparation planning continued for the next six months.

The pilot project began three months into the rectification project preparation phase. Refinements continued throughout the next two months until the pilot was completed.

Following the pilot project, the remainder of the rectification process commenced. Data was "frozen" in batches in the legacy production environment, rectified, quality assurance checked and returned to the production datastore after passing quality assurance checking. This process continued until the entire NIPSCO AutoCAD DWG files were rectified and placed back into the legacy production environment. 


\subsection{Data Migration}

The data migration and data rectification processes required significantly more effort than could be accomplished by the GIS project team while developing the AEDR, and therefore was packaged together with the data rectification work as an external contracted component.

Data migration entailed moving NIPSCO's source land, gas and electric data from the legacy format into NIPSCO's new ArcGIS/ArcFM data model. More specifically, graphic and attribute data from the AutoCAD files, partially replicated Microsoft Access database and the Electric Distribution Facility Service (EDFS) mainframe DB2 database was loaded into NIPSCO's ArcGIS/ArcFM data model to support NIPSCO's future ArcGIS/ArcFM system and the use of the data within that system.

NIPSCO worked with its software and service supplier partners to design, develop, and configure the ArcGIS/ArcFM system and the associated data model. Following completion of the data model development and ArcGIS/ArcFM configuration work, NIPSCO's existing source data was migrated to the ArcGIS/ArcFM geodatabase format.

The fundamental deliverables associated with the data migration effort was NIPSCO's land, electric, and gas data in ArcGIS/ArcFM geodatabase format.

The challenge was to determine which of the two data stores (AutoCAD or EDFS) contained the most accurate information, eliminate the duplicate data, report on mismatched information for future investigation and cleanup, and migrate the accurate data to the target.

The EDFS served as a consolidated and centralized repository for pole card data, transformer life record card data, underground electric facilities data, street light location and dusk-to-dawn lighting record cards. EDFS' ability to interface with the customer information, material and supply, material and labor estimating, wood pole treatment and general ledger account validation systems eliminated numerous entries of duplicate information.

Operations as well as several user departments had access to this system for preparing monthly and/or annual property unit reports, transformer history and test data, pole treatment and reinforcement data and environmental documentation. User departments benefiting from EDFS include: Purchasing, Material Services, Environmental, Transformer Shop, Rate Base, General Accounting, Resource Planning and Engineering.

Data migration specifications were developed using a migration matrix (spreadsheet) of source to target instructions for the data. Finely detailed specifications were developed for derived data processes which are those processes that were not simply a one for one move, but involved merging and or conversion of the data into a different format. 
Miscellaneous migration specifications such as device edge splitting requirements, geometry alterations and secondary conductor migration were developed in addition to the migration matrix and included with each delivery of data to the migration vendor.

NIPSCO worked with the data migration vendor to refine and demonstrate the suitability of the data migration specifications and processes through a graduated approach whereby each delivery of migrated data contained substantially more features until the final two deliveries contained the entire dataset.

Delivered migrated data was subjected to a series of automated and interactive quality acceptance tests. Acceptance or rejection of the delivered data was determined based upon the results of the acceptance tests. The testing regime combined $100 \%$ examination relative to certain delivery characteristics and examination of a random sample of the delivered data relative to certain other delivery characteristics. Migration deliveries demonstrating a clear failure to meet the objectives of the delivery were rejected. Requirements for improvement of the migration process that were identified through review of any preceding delivery that were not demonstrated within a subsequent delivery were rejected.

NIPSCO utilized the following criteria in determining the acceptability of the final full data migration delivery:

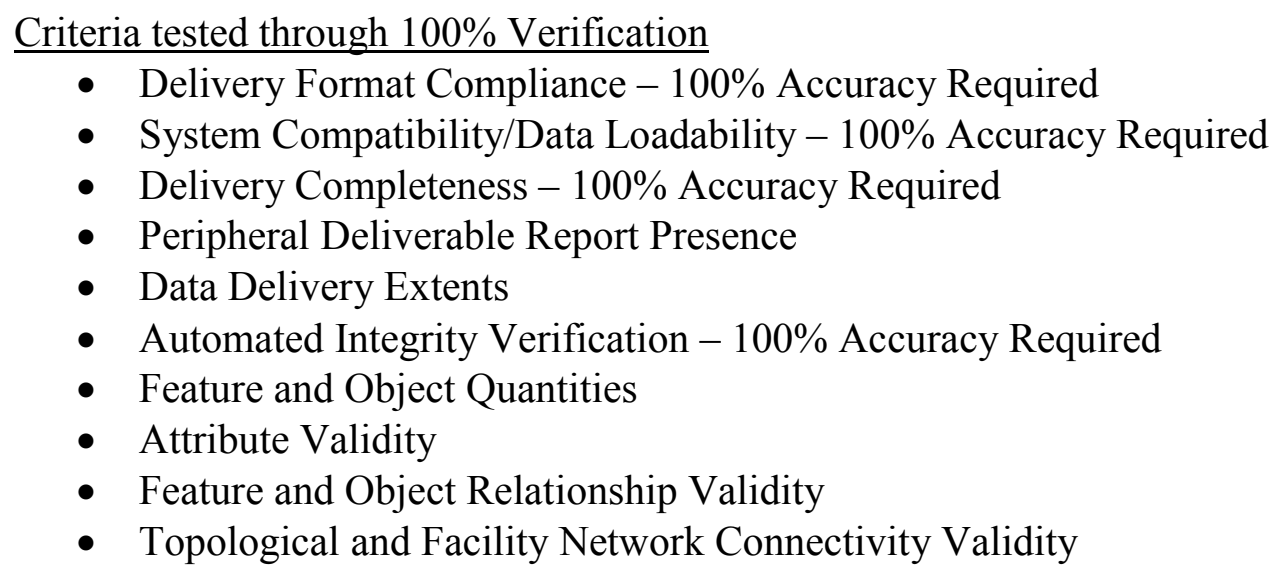

The vendor was required to segment, aggregate, associate, and / or manipulate the data in order to create appropriately segmented, joined, related, connected, and populated GIS features and objects.

A highly detailed data migration plan was developed and followed and the project team was required to review and update the Plan prior to it's execution for each migration delivery. The Plan provided direction and helped to ensure that no steps were missed during the complex preparation of the data and environment for each migration delivery. A quality assurance plan checklist was also developed to guide the quality assurance process, ensuring that no steps were missed. The checklist also served as a timing mechanism for each subsequent quality assurance process as the timeframes were recorded on the Quality Assurance Plan spreadsheet. Quality assurance team members 
were required to record their start and stop times and note any comments on the sheet. This Plan proved crucial in determining subsequent quality assurance timeframes as part of the total implementation process duration.

It was determined that, the segmenting, aggregating, associating, and / or manipulation of the data in order to create appropriately segmented, joined, related, connected, and populated GIS features and objects along with some fairly simple migration proved too challenging for the migration vendor. In order to complete the migration and implement the AEDR, NIPSCO internal resources wrote in excess of forty migration routines that migrated NIPSCO's data where the migration vendor was unable.

\subsection{ArcFM / ESRI Configuration}

ArcFM and ESRI Configuration consisted of configuring a combination of the out-of-thebox and custom ArcFM Properties, ArcFM Snapping, ESRI Relationship Rules, and ESRI Connectivity Rules. While this exercise may seem trivial on the surface, the time invested in these tasks has an enormous impact on the end user experience as well as the quality and integrity of the data managed by the systems. The participants in the base configuration included core team members, end user representatives, and consulting and product vendors who had specialized knowledge in the conceptual and practical implementation of the configuration.

The ArcFM Properties Manager, an extension to the ESRI ArcCatalog application was used to configure ArcFM.

The baseline ArcFM Properties requirements were captured using a two step approach. First, the configuration that constituted end user preferences were captured during a series of interview sessions with key end users. Secondly, baseline configuration included configuring the out-of-the-box ArcFM tools and AutoUpdaters to run on the NIPSCO data model. This was accomplished by using the published ArcFM configuration help files. Once captured, this information was exported and saved in standard XML files. When needed, XSLT is applied to the XML files to arrange them into an easy to read format.

Once the base configuration was completed, the configuration for all custom software components was applied to the geodatabase via the ArcFM Properties. Each of the custom installations includes some form of an Operations Guide which details the custom configuration for both the Model Names and the component assignment. These are applied in the same manner as the base configuration using the ArcFM Properties tools.

\subsection{AEDR Customization}

The AEDR was highly customized in that the entire Electric Facility Distribution Services (EDFS) application for managing NIPSCO's electric assets was integrated into the AEDR application. Additionally, other customizations were conducted on the AEDR thereby fortifying the AEDR to the rich, user friendly tool that adds the depth to the value 
a GIS brings to NIPSCO. The customizations are discussed in two parts; Section 2.7.1 EDFS GIS Functionality and Section 2.7.2 Non-EDFS GIS Functionality.

\subsubsection{EDFS GIS Functionality}

The integration of EDFS functionality into the new ArcGIS/ArcFM environment was accomplished with a blend of data model and database updates, customization to ArcFM, and a custom Stand Alone Geodatabase Editor (SAGE), which exists independently of the GIS applications. The goals of this implementation included both the replacement of the EDFS functionality as well as the enhancement of both the technology and the business processes to provide a more efficient and effective solution for all concerned users of the system.

The following components were used / designed to integrate the EDFS functionality into the new AEDR/ArcFM system:

- GIS Data model changes

- EDFS Data Migration

- ArcFM Auto Updaters

- Batch Data Management and Reporting Applications

- External System Interfaces

- SAGE Architecture

- SAGE Authentication and Authorization

- SAGE Data Model

- SAGE Screen Design

- SAGE Reports

The integration objectives included the creation of functionality in the new ArcGIS / ArcFM system to satisfy all business needs related to the current EDFS system:

- Lifecycle management of Units of Property including Transformers, Regulators, and Capacitors

- Lifecycle management of all other standard overhead and underground assets including Poles, Pads, Vaults, Assemblies, Protective Devices, Conductor, and Lighting

- Current and legacy reporting on assets for both internal and external entities

- Interfaces to other NIPSCO systems including MAPPS (Materials, Purchasing and Accounts Payable), MLOG (Compatible Units), General Ledger, CIS (Customer Information System), and Tax Department.

To improve the efficiency and effectiveness of the manner in which the data is accessed the project team focused on:

- Integrating asset data storage into the GIS

- Integrating asset data management into ArcFM (installation, removal, retirement, etc)

- Providing non-GIS data access to users who do not rely on the spatial aspect of the data

- Removal of unnecessary batch reporting while maintaining business value, and 
- Improving workflow of targeted users by making data updates more efficient and straightforward by leveraging more modern technologies.

The NIPSCO Electric Distribution Facilities System (EDFS) was a stand-alone asset management application that managed the utility's electric assets including transformers, regulators, capacitors, primary, secondary, and service conductor, poles, pads, streetlights, traffic lights, and miscellaneous data surrounding these assets.

EDFS was responsible for managing the life cycle of these assets from the time of their acquisition through retirement or scrapping. This life cycle can include multiple installations, retirement, condemnation, sale, and / or lease. In addition to managing these assets, EDFS was responsible for generating many reports based on the current and / or historical states of the assets. These reports were used by management for the assessment of the current assets as well as tax reporting purposes.

There was no integration between EDFS and NIPSCO's mapping tool, a custom CADbased application called Outfield. During migration, EDFS data records were manually matched to Outfield geographical records based on unique IDs.

An extensive review of the proposed NIPSCO data model was conducted to identify the changes that were required to implement the EDFS functionality within the AEDR.

Much of the EDFS functionality was handled by out-of-the-box (OOTB) ArcFM tools within the AEDR including the ArcFM Attribute Editor. This tool allows the users to update the various EDFS records within the AEDR but does not handle any custom validation and / or field population. To accomplish these automated tasks, several simple ArcFM Auto Updaters (AUs) were created. Additionally, several ArcFM tools were developed to assist with the users' edits within the GIS and several batch applications were written to manage the EDFS data that are scheduled to run via the Windows scheduler. The majority of the NIPSCO reports are generated in a batch fashion.

The AEDR has extensive batch processing, to name a few:

- Interface Management

- Structure to Conductor Join

- Release SDE Lock

- Domain Synchronization

- Asset Statistics

- Session Reporter

- Batch Reconcile and Post

- Batch Reconcile and Compress

- File Archiver

- Condemned Asset Cleanup

- On Demand Reports Cleanup

- Street Centerline Intersection Creator 
The SAGE application uses the .Net's built in Windows Authentication modules. This allows the users to connect to the web application without accessing a logon screen. The user's authentication credentials are read from the client machine via the connecting http request and the authentication transaction appears seamless to the user.

The user's username is extracted from the security credentials and is used to query the SAGE security tables to access the user's authorization role. The authorization role is used to grant or deny the user access to each area of functionality within the SAGE application.

The SAGE application requires a small database to manage the non-GIS data aspects of the application. These areas include the authorization model of the SAGE application, various tables relating to the nightly processing of the MAPPS transactions, and a join table that relates the distribution reference number of poles, pads, and pedestals to conductors (overhead and underground primary and secondary) for reporting purposes. This database is managed as a separate database within the same SQL Server instance as the GIS.

The SAGE Reports are modeled after the EDFS reports in regard to the data they display and the layout. The data for the reports were gathered by using SQL queries against the SAGE multi-versioned view. The queries and logic for the reports were derived from the original EDFS design specifications. The data was converted into an XML format using .Net tools and then transformed into the desired format by using XSLT stylesheets.

The batch reports that are available in the AEDR system include:

- Request for Authority to Condemn Transformers and Regulators

- Line-Transformer / Voltage-Regulator Company Use Report

- FERC For-1 Report of Line Transformers

- Report Showing Transformers and Poles

- Annual Count of Transformers

- Annual Count of Transformers by County

- Transformer Failure Report

- Temporary and Inactive Transformer and Regulator Installations

- Transformer \& Regulator Stores Item Number Description Report

- Transformers In Stock Inventory Report

- Annual Count of Capacitors

- Capacitor Failure Report

- Capacitor Stores Item Number Description Report

- Capacitors In-Stock Inventory Report

- Securities and Exchange Commission Form 10K

SAGE screen design is organized into functional areas and is driven by the drop down menus at the top of the screen. A sample screen is shown in Figure 2-4. 


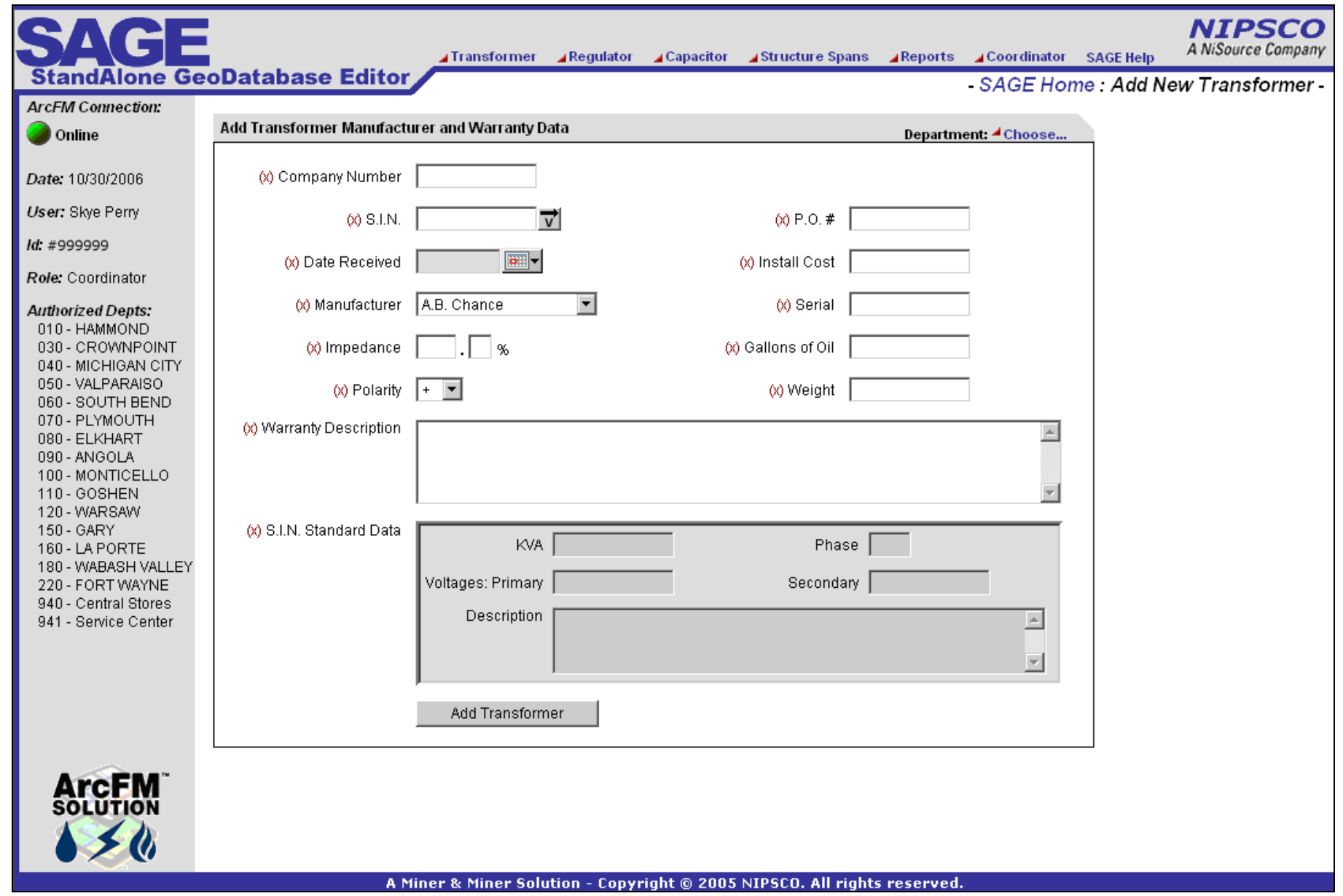

Figure 2-4. Add Transformer Manufacturer and Warranty Data Screen

Query, Reporting and Edit capabilities are extensive, to name a few:

- Query by Company Number / Serial Number

- Query by Location

- Edit Existing Transformer \& Capacitor Manufacturer and Warranty Data

- Transformer \& Capacitor Install

- Transformer \& Capacitor Remove

- Transformer \& Capacitor Delete

- Transfer Property (from one location to another)

- Transformer Status

- Transformer Replacement

- Add New Capacitor

- Structure Span Lengths Query

- Structure Span Lengths Add / Edit / Delete

- Sessions Inquiry Report

- Capital Assets for Sale Report

- Pole / Pad Mounting Inquiry Report

- PadMount Inspection Form Report 
- Batch Reporting Screen

- Coordinator Dashboard

- SAGE Security Report

- Add New SAGE User

- Manage SAGE Roles

- Unique Distribution Reference Administration

- Reset Condemnations

- Repair SDE Lock

\subsubsection{Non-EDFS GIS Functionality}

Various software requirements were gathered to enhance the ArcGIS / ArcFM functionality to make it better meet the needs of the NIPSCO end users. Much of this was driven from the review of required functionality within the previous AutoCAD Outfield system. Other areas were implemented to streamline the user's interaction with the system, ensure data integrity, or to auto-populate data required by a new system interface. Some customizations simply provide a more efficient manner in which to enable an existing business process.

This is a partial list of AutoUpdaters, Edit Tasks, Subtasks, and tools that were created outside the scope of the EDFS replacement:

- Copy Value to Related Object

- Cross Over Arc

- Session Manager Do Not Post Subtask

- Field Concatenation AU

- LOA Number AU

- Network Edge Split at Tap Point

- Normal Position Symbology AU

- Prevent Delete if Object has Related Objects

- Reference Features Remove Button

- Feature Offset Edit Task

- Unique Distribution Reference AU

- FeederAll Open Point Deletion AU

- Transformer Lead Edit Task

- Delete Attached Transformer Lead

- Abandon Gas Features

- Place and Relate Gas Main Edit Task

- Retrieve ObjectID of Closest Gas Main

- CP Section - Corrosion Control Number

- CP Section-Maintenance Tool

- Gas Main Pipe Change

- Emergency Valve Indicator

- Valve Number AU

- Custom Identify Tool

- Custom Structure Relate 
- Custom QAQC Tools:

- Delete Report

- All Edits Report

- New Records

- Updates

- All Edits Options

- All Edits Tools

- Custom Login

- Citrix Publishing - ArcLauncher

\subsubsection{GIS to CAD Conversion}

Since engineers required NIPSCO data in the form of an AutoCAD drawing file (DWG), a CAD Conversion tool was required that would export GIS data from the NIPSCO Geodatabase to AutoCAD 2000 DWG files. A set of pre-existing, non-productized tools developed by Telvent Miner \& Miner running on top of Safe Software's productized Feature Manipulation Engine (FME) became the solution and "CAD Converter" was configured to generate the AutoCAD files. Eventually, the CAD Converter tool was replaced with Ptarmigan CAD Converter when it became necessary to export unusually small files to the ABB CADOPS interface landbase.

Bi-monthly, the Ptarmigan CAD Converter tool is used to generate a complete set of DWG files from the geodatabase. These files are restored to a share accessed by the engineering department. Ptarmigan CAD Converter is also used to process third party requests to produce maps in an AutoCAD DWF format.

The remaining customizations and their details can be found in the "Validation Integration - Results of Future Architecture Implementation" NREL Report No. SR-58143432”.

\subsection{AEDR Interfaces}

Maintaining the current system interconnections under the AEDR implementation required legacy interfaces to be preserved or re-written if necessary to minimize disruption of current business processing. Some of the interfaces required minimal effort and others were completely re-written.

\subsubsection{Facility Browser Interface}

A legacy Facility "Browser" provided web-based access to NIPSCO's facility data for a large volume of back office users. The Facility Browser also provided limited editing capability so it was not really a true "viewer / browser" application, and it used AutoDesk MapGuide as its mapping engine. The Facility Browser application was re-written to become a true viewing application and to utilize ESRI's ArcIMS as the mapping engine and ArcSDE for the spatial data component.

Figure 2-5 shows the main user interface for the new Facility Browser: 


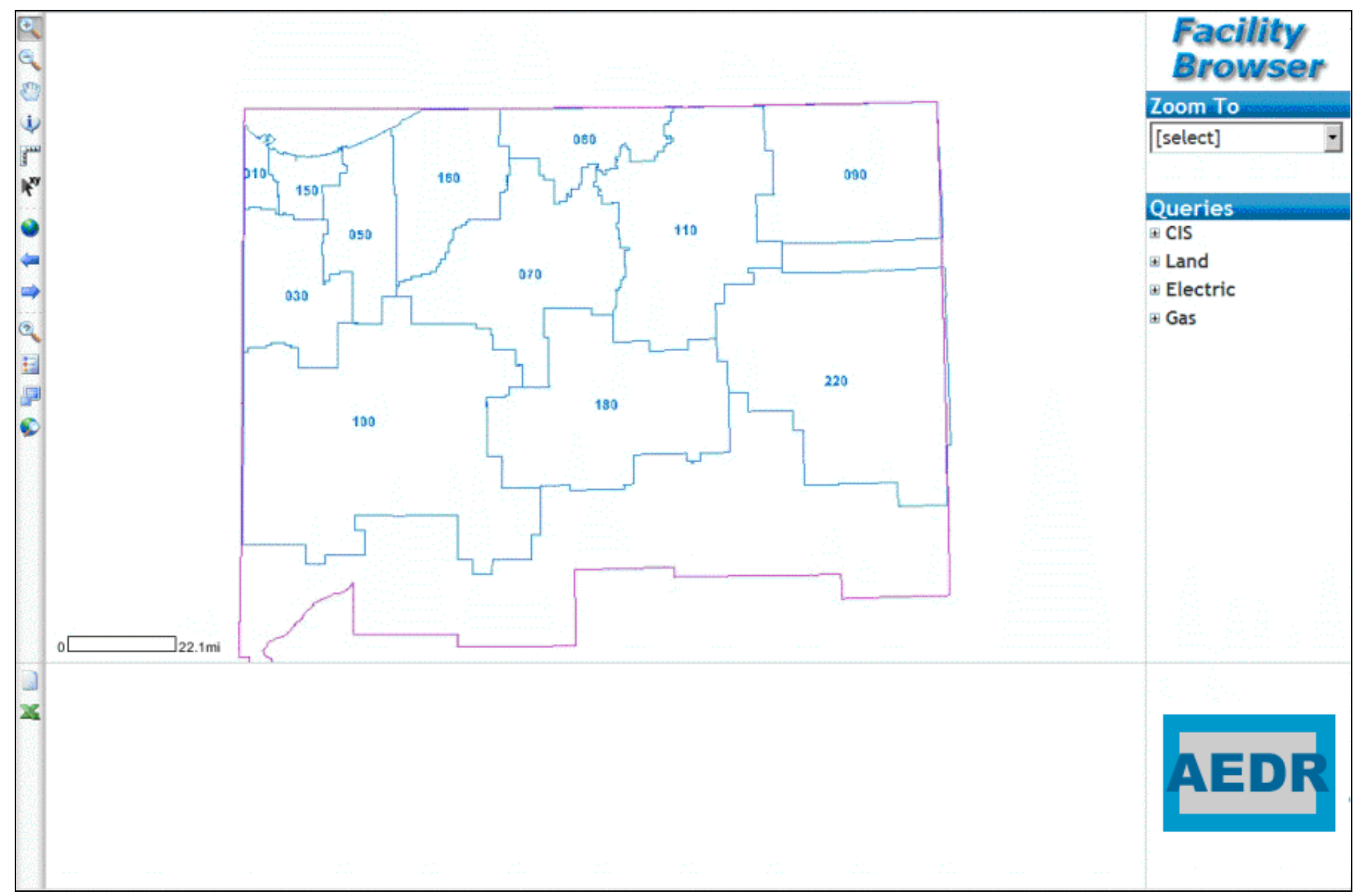

Figure 2-5. Facility Browser main user interface

\subsubsection{Field Browser Interface}

A legacy Field Browser in contrast, was originally designed as a field viewer, however many back office users latched on to its' simplicity and convenience of use. The legacy Field Browser application was a self contained installation of NIPSCO data and AutoDesk's unlicensed VoloView application. The Field Browser data was updated monthly by a manual extract of data and manual load of that data onto the Field Browser laptops and back office desktops.

The new Field Browser solution uses ESRI's unlicensed ArcReader application and the same monthly manual data extraction and loading on each laptop (or back office user computer) continues. It remains a true viewer application and back office users remain a significant user base.

Figure 2-6 shows the main interface for the new Field Browser. 


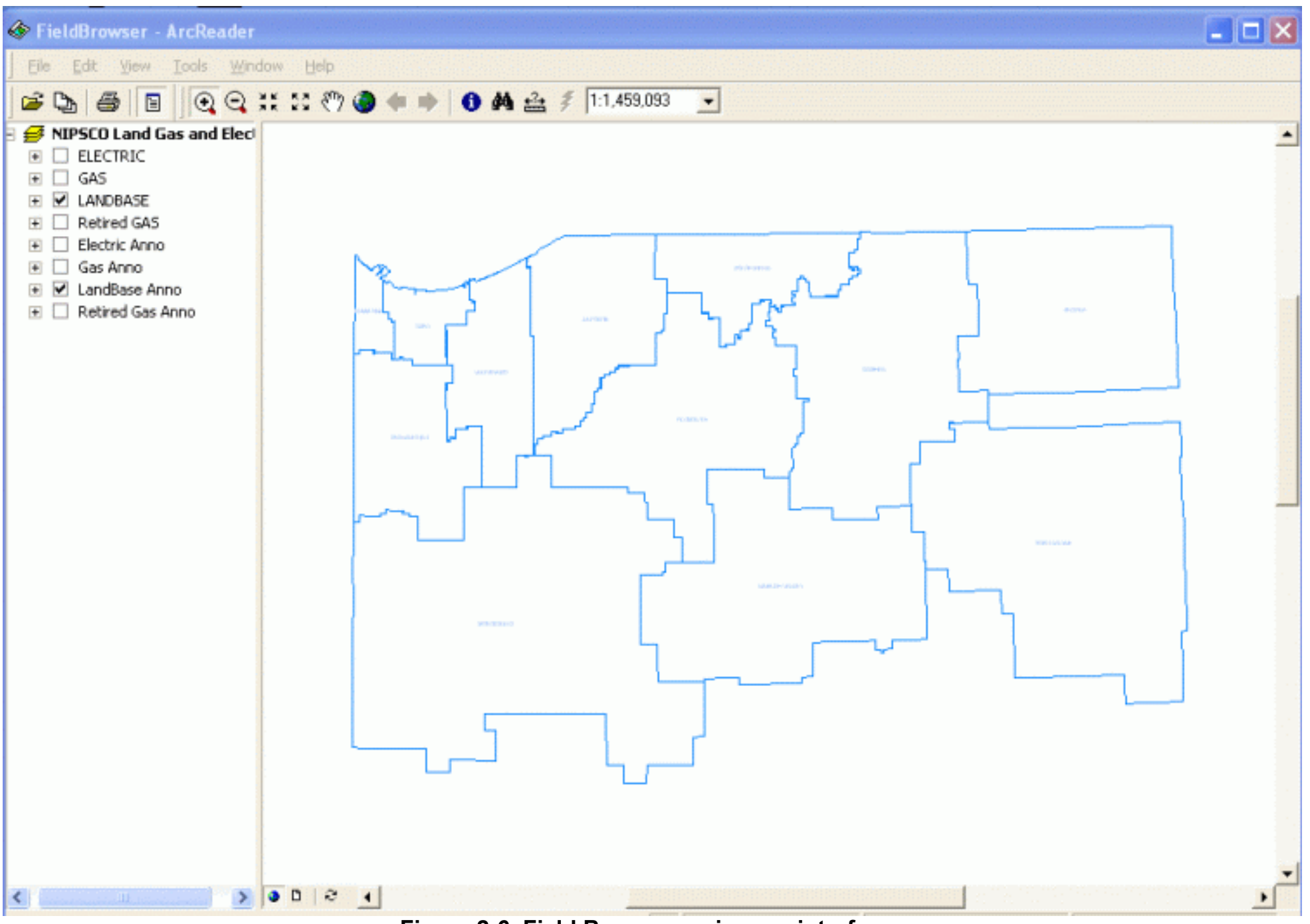

Figure 2-6. Field Browser main user interface

\subsubsection{SynerGEE Interface}

Legacy DataPrep was a component of an interface between the SynerGEE application and the legacy Outfield mapping system. Legacy DataPrep exported gas system data from NIPSCO's database of AutoCAD drawings as comma delimited text files. A second application called MiddleLink read these text files to populate a SynerGEE database. Finally, the SynerGEE application used the database for gas system flow \& pressure calculations.

Because the facility data is now stored and maintained in a different technology platform, the legacy DataPrep component to the SynerGEE interface was re-written. The new custom DataPrep application creates the same text files in the same format as the legacy DataPrep application. The DataPrep export procedure was required to yield data values identical to the original Outfield data in order to support the target applications. However, instead of exporting the data from tiled AutoCAD drawings, the new application exports the data from a seamless ESRI Geodatabase. 
The DataPrep application is built as an ArcMap extension, which adds functionality to the out-of-the-box tools. The DataPrep extension adds a toolbar to the ArcMap graphic user interface, with a single command button. The button launches a wizard that guides the user through the export process. A number of configuration settings necessary for the DataPrep Application are configurable via an XML file. The custom DataPrep adds more functionality and independence to the original process.

\subsubsection{CADOPS and FeederALL Interfaces}

Electric outage management and electric load studies are discussed together as NIPSCO uses both ABB CADOPS and ABB FeederAll and they are both very similar interfaces to AEDR.

For outage management in the electric distribution system, NIPSCO uses ABB CADOPS running on top of an Oracle database. Previously, NIPSCO used a tool called ABB OUT to export facility data from legacy Outfield into a comma-delimited file. Operations then used Oracle SQL Loader to load the data from the comma-delimited file into the CADOPS Oracle tables where custom SQL scripts were then run to update additional information.

For engineering analysis in the electric distribution system, NIPSCO uses ABB FeederAll. In the legacy system, a copy of the CADOPS comma-delimited file was used to supply the data for FeederAll. Oracle SQL Loader was used to load the data from the comma-delimited file into the FeederAll Oracle tables where custom SQL scripts were then run to update additional information. Next, an ABB process was used to transfer the Oracle data into a Microsoft Access database, which supports the FeederAll application. Additional hand entry of data within the MSAccess database was necessary to support the analysis aspects of FeederAll.

To support the new interface, facility features and the network hierarchy are exported into the required comma-delimited file formats. This is accomplished using ArcFM Network Adapter to select either a section or a feeder of data for export. The export process uses the XML produced by the Network Adapter API and transforms it to create the aforementioned comma-delimited formats for CADOPS and FeederAll. Only facility network geometry and attributes are exported - no annotation is exported for facility information.

The Figure 2-7 diagram illustrates the new interface: 


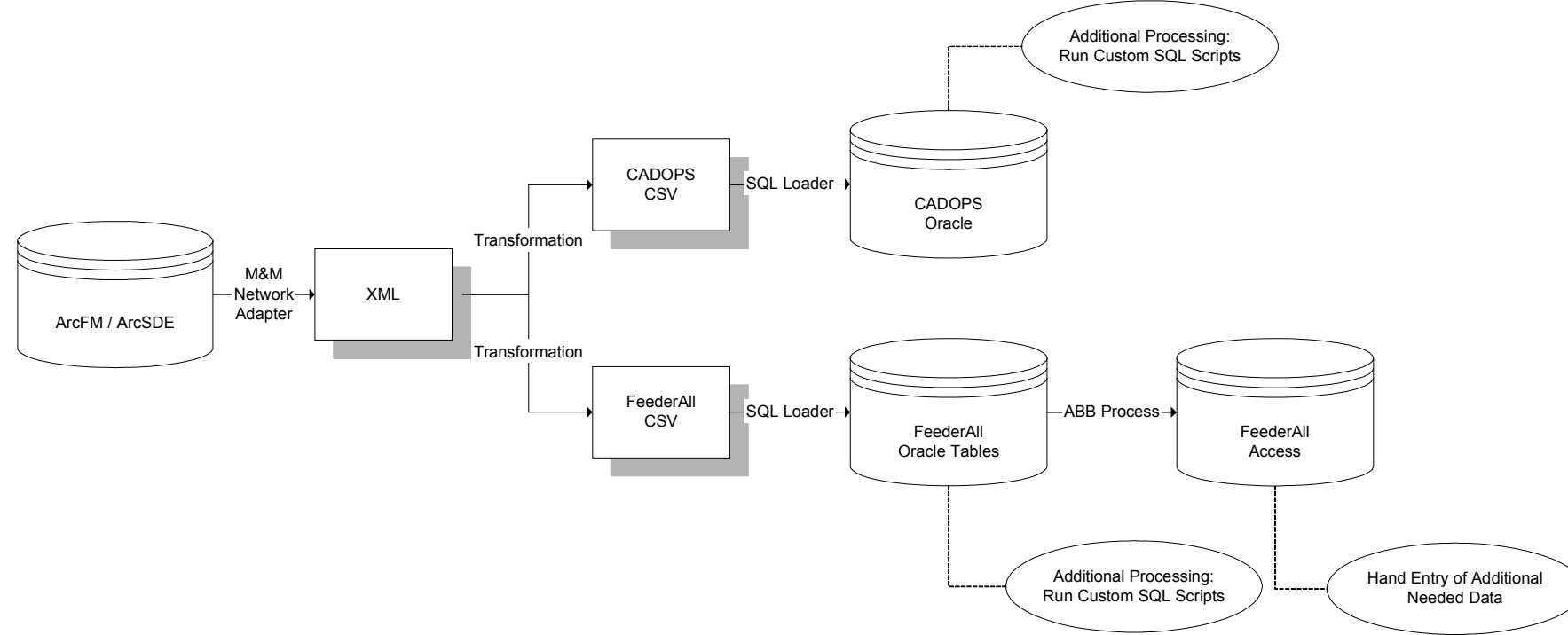

Figure 2-7. CADOPS/FeederAll interface diagram using Network Adapter to produce the XML file

When the XML is transformed into the CADOPS and FeederAll comma-separated-value (CSV) files, the new process matches the process that existed previously.

\subsubsection{Material, Accounts Payable and Purchasing System (MAPPS) Interface}

Several interfaces exist between the AEDR and the MAPPS. These interfaces were rewritten:

- Stores Item Number Description

- Nightly Transactions

- Near Real Time Asset Push

\subsubsection{Customer Information System (CIS) Interface}

Several interfaces exist between the AEDR and the CIS. These interfaces were rewritten:

- CIS Site

- CIS Installed Service

- Transformer Install and Removal

- Support Structure and Padmount Installation

- Installed Service Coordinate Update

- CIS Account Summary

- CIS Service Address Summary

- CIS Life Support Summary 


\subsubsection{General Ledger Interface}

AEDR work order validation is made possible by an interface to the General Ledger shown in Figure 2-8.

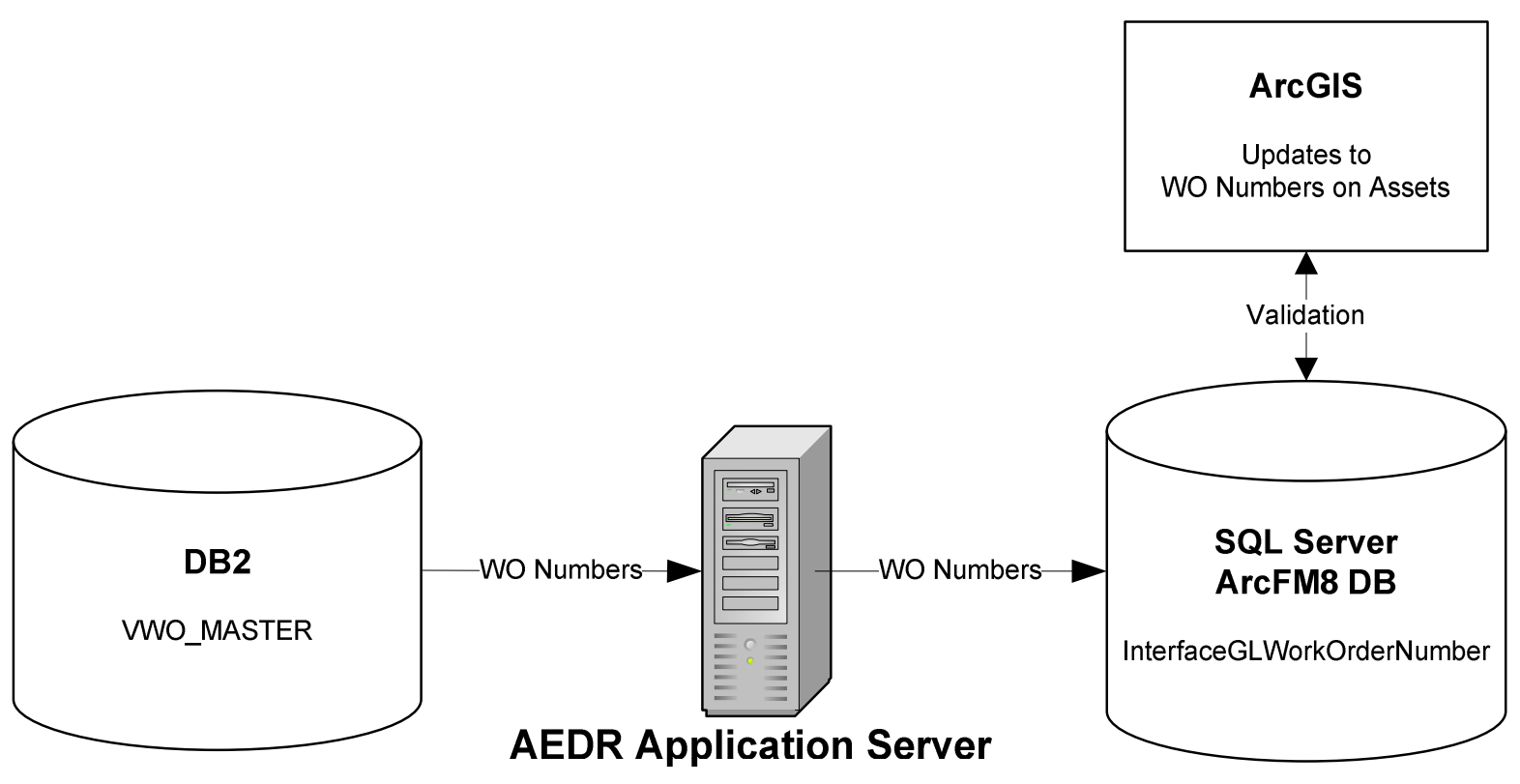

Figure 2-8. Work order validation process

\subsubsection{Engineering Accounts Information File (EAIF) Interface (Indirect)}

The EAIF application allows engineers to perform load analysis on a specific transformer based on the properties of the transformer combined with the customer consumption data tracked in the CIS. Previously, the EAIF application directly accessed the EDFS tables to get the transformer unit and installation data. In the new AEDR system, this information is managed within the GIS. When transformers are created, installed, and removed within the GIS, this information is now passed to the EAIF via the CIS interface on a regular basis. EAIF was updated to read the transformer data directly from the CIS DB2 tables in place of the EDFS tables. This is an indirect interface because the data is ported through another interface (CIS).

\subsubsection{Material and Labor Online Guide (MLOG) Interface}

The Material and Labor Online Guide (MLOG) system manages compatible unit / assembly numbers within NIPSCO. This interface was not rewritten, just reestablished in the new GIS. In the future, the AEDR will incorporate Telvent Miner \& Miner's Designer and a Work Management process that will eliminate the need for this interface. 


\subsection{System Performance Evaluation / Stress Testing}

In order to maximize the usability of AEDR, NIPSCO tuned the system for maximum performance. Performance was defined as the application's impact on productivity.

Therefore, the goal was to ensure a system performance level that complimented the end user workflow.

NIPSCO used the following strategies to achieve adequate system performance:

- Implement basic performance configurations prior to rollout.

- Run tests to evaluate the performance of the system and to identify potential performance enhancements prior to rollout.

- Based on performance testing, fine-tune the system prior to rollout.

- Conduct regular performance maintenance on the production system.

In tandem with system performance testing, performance testing of the ArcSDE / SQL Server database server was conducted. Nightly compression and index rebuilds are executed on the database to maintain performance in the production environment.

\subsection{AEDR Online Help Documentation}

A comprehensive and centralized online help documentation system was needed to provide a single source for all AEDR users to access for information and operational instructions.

A customized web-based solution is hosted at an intranet web address and contains sections for the following five main AEDR components:

- ArcEditor

- ArcView

- SAGE

- Facility Browser

- Field Browser

Additionally, it was decided to include web-based authoring and management tools in the solution so that updates to the Help Content could be deployed by users with no

knowledge of html, JavaScript, the web server, etc. This allows a standard administrator (admin) user to create and deploy help content in a seamless environment.

Figure 2-9 shows the main user interface to the help system. 


\begin{tabular}{|c|c|}
\hline Contents Index Search Favorites & AEDRONLINE HELP SYSTEM \\
\hline $\begin{array}{l}\text { CAEDROnline Help } \\
\text { Overview } \\
\text { ArcView Help } \\
\text { Facility Browser Help } \\
\text { Field Browser Help } \\
\text { SAGE Help }\end{array}$ & 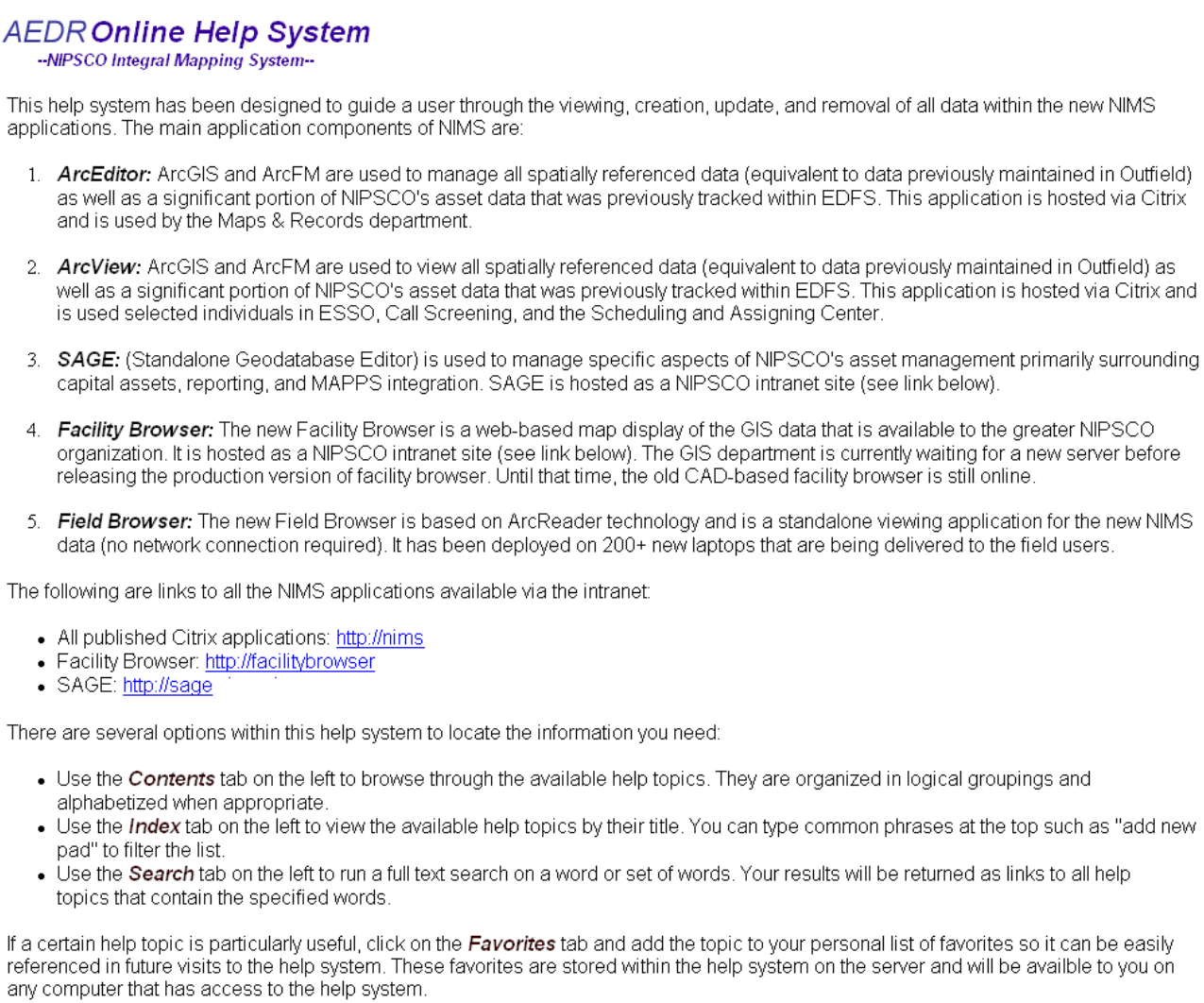 \\
\hline
\end{tabular}

Figure 2-9.

\subsection{Training}

At the beginning of the project we understood that user training would present a challenge not only because of the logistics of a widely distributed workforce, but also because of counsel given by contractors experienced working on large scale GIS projects. Users unfamiliar with GIS concepts would require intensive training and practice before being turned loose in the production environment. We were determined to equip our users with as much knowledge and experience as possible prior to production implementation.

The training component required a great deal of organization and commitment both from the GIS project team and also the users. The user community was further challenged by the streamlined workforce. This presented few options for covering the business activities during training.

The GIS project team was trained from both a technical and functional perspective, while training for the end-users was purely functional. NIPSCO adopted the "Train the Trainer" concept of training GIS project team members to assist in training the end-users. 
The training effort of planning, preparing and organizing began eight months in advance of onsite training.

Telvent Miner \& Miner were contracted to perform the user training and this worked well for NIPSCO. The project implementation timeframe was lengthy and during the gap between training and production implementation, users were required to practice with real-life business case scripts four hours per day for six weeks prior to production implementation of the AEDR. Members of the GIS project team assisted the users with training issues. The extended practice sessions paid off, most users were ready for production activities when the AEDR was installed into production.

\subsection{Help Desk}

Critical to providing end-user support post-implementation was the Help Desk function provided in part by the user community. Record Department record clerks were the core GIS users, responsible for maintaining the facility data through the closeout of work orders. The Records Department Help Desk supports a dual function in that they are responsible for reconciling, posting and resolving conflicts through Session Manager. By instituting a user-based Help Desk function, they were able to become power users and assist the record clerks often without involving the GIS project team.

\subsection{Implementation}

The Implementation Plan / Schedule governed the production implementation of the AEDR. Over the course of two months preceding the implementation, preparations were underway and the Implementation Plan was constantly being refined.

Once the data was delivered to the migration vendor, record clerk training and final integration testing was performed. The vendor migration process spanned sixteen consecutive days and when the data was returned to NIPSCO, it took several days to load the data into the migration instances to prepare it for both automated and interactive quality assurance checking and simultaneously prepare a separate migration build instance for the remaining migration of data that the NIPSCO team was required to perform.

Over the next three weeks, the data was programmatically and interactively quality assurance checked, data cleanup scripts were run, the geodatabase was configured, ArcFM's FeederManager was run and the separate migration build (NIPSCO's GIS team migration) was quality assurance checked. For two days following the completion of the quality assurance checking process, bulk loads and miscellaneous batch processes were run on the data to complete the preparation of data for production. Subsequently, we opened up production for a small group of users to ensure that any major problems could be addressed before the entire user base began using AEDR in production. The small group used the AEDR for two consecutive days and when no problems were found, the full production environment was opened to the entire user base. Over the next three weeks, GIS project team members were assigned a production support stand-by role to assist in any major system problems. However, no major problems surfaced and the team mostly addressed individual user training issues. 


\subsection{Wind Generation Integration Study}

In a joint collaboration with the National Renewable Energy Laboratory (NREL), the project team performed a feasibility study for potential wind farm integration within the NIPSCO service territory.

Utilizing NREL's graphical Renewable Planning Model (RPM) application as a starting point, this tool helped identify three wind generation sites located within NIPSCO's service territory. From the modeling perspective, each of these three sites identified in Figure 2-10 offers the feasibility of renewable energy production at a utility scale.

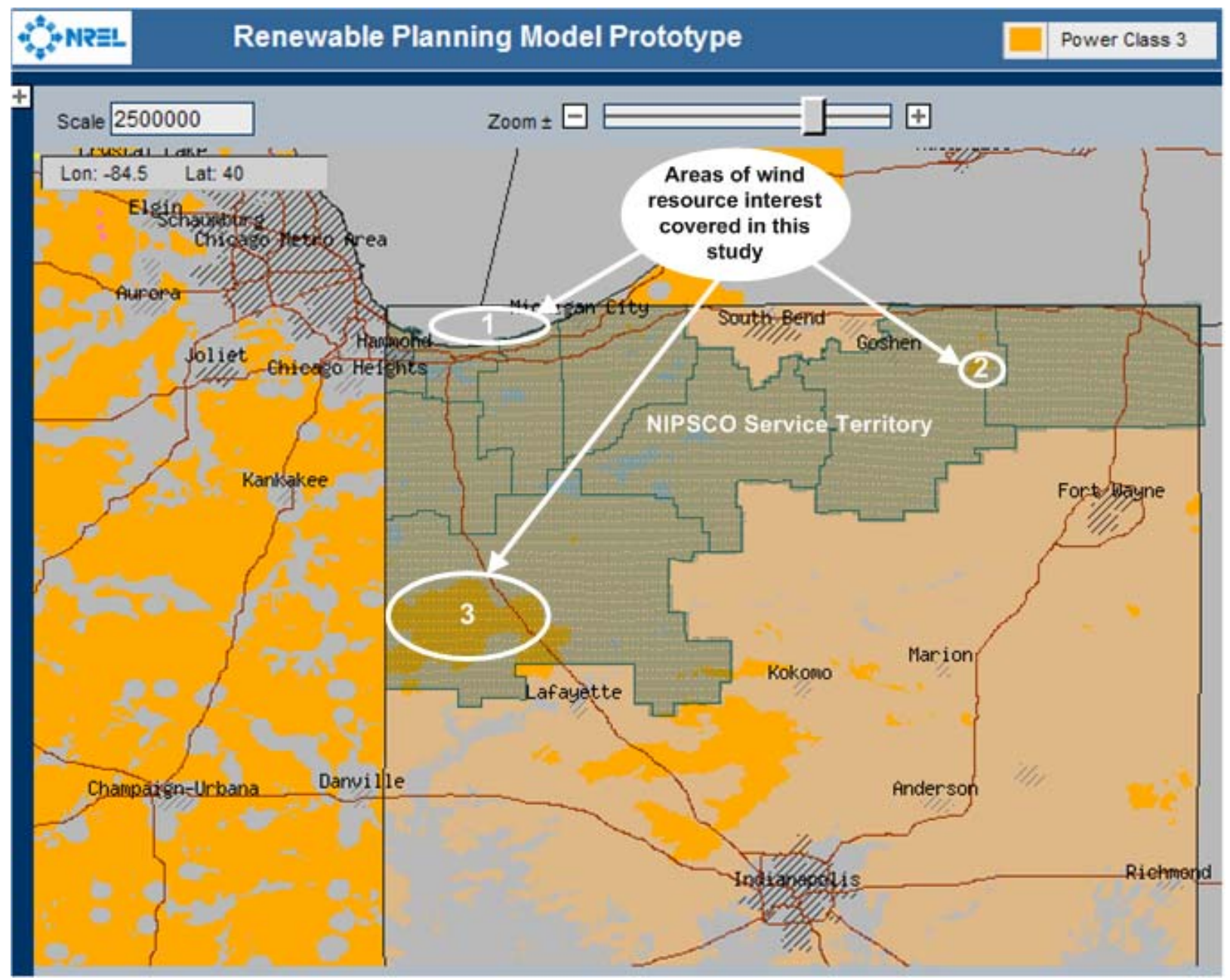

Figure 2-10.

The RPM application links NREL and NIPSCO's Geographic Information Systems (GIS) data to map company facilities and estimated wind generated electric loads that could be potentially integrated into NIPSCO's electric transmission grid. This GIS data was overlaid on top of the NREL's GIS data to identify wind resources, map grid locations, and land usage via satellite images. Further details of this study can be accessed in the "NIPSCO Wind Generation Integration Study" contained within the "Validation 
Integration - Results of Future Architecture Implementation” NREL Report No. SR-58143432.

\subsection{ESRI EGUG Presentation}

The customization to include NIPSCO's asset integration into the AEDR was presented at ESRI's 2007 Electric \& Gas Users Group Conference under the title "Managing Assets in Your GIS - History in the Making". Figure 2-11 shows the end of the presentation which can be found in its entirety on the ESRI Web site in the 2007 Proceedings link: http://gis.esri.com/library/userconf/egug2007/index.html

\section{Managing Assets in Your GIS}

SSP

Questions \& Comments

Welcomed

Diane Buche - NIPSCO / NiSource dlbuche@nisource.com

Skye Perry - SSP Innovations, LLC skye.perry@sspinnovations.com

This work was supported under subcontract NAD-5-33652-01

(Technical Manager Thomas_Basso@nrel.gov) with the

National Renewable Energy Laboratory managed by the Midwest Research Institute for the US DOE under contract

Number DE-AC36-99G010337

Figure 2-11. 


\section{Conclusion}

Modernizing NIPSCO's electric-gas utility infrastructure by implementing a Geographic Information System (GIS) technology has resulted in the benefits expected for both NIPSCO and its customers. Distinct and not readily interoperable technologies have been assimilated into a single state-of-the-art GIS with efficient and far-reaching information dissemination capabilities.

Figure 3-1 demonstrates the AEDR has reached expected goals of implementing a solution for:

- managing all of NIPSCO's gas, electric and landbase objects in one database

- data integrity improvement through rectification, data cleanup, migration processes and additional validation

- Integrated gas and electric load studies through the use of both custom and vendor solutions, and

- has maintained or improved the interfaces necessary to provide a complete solution. 


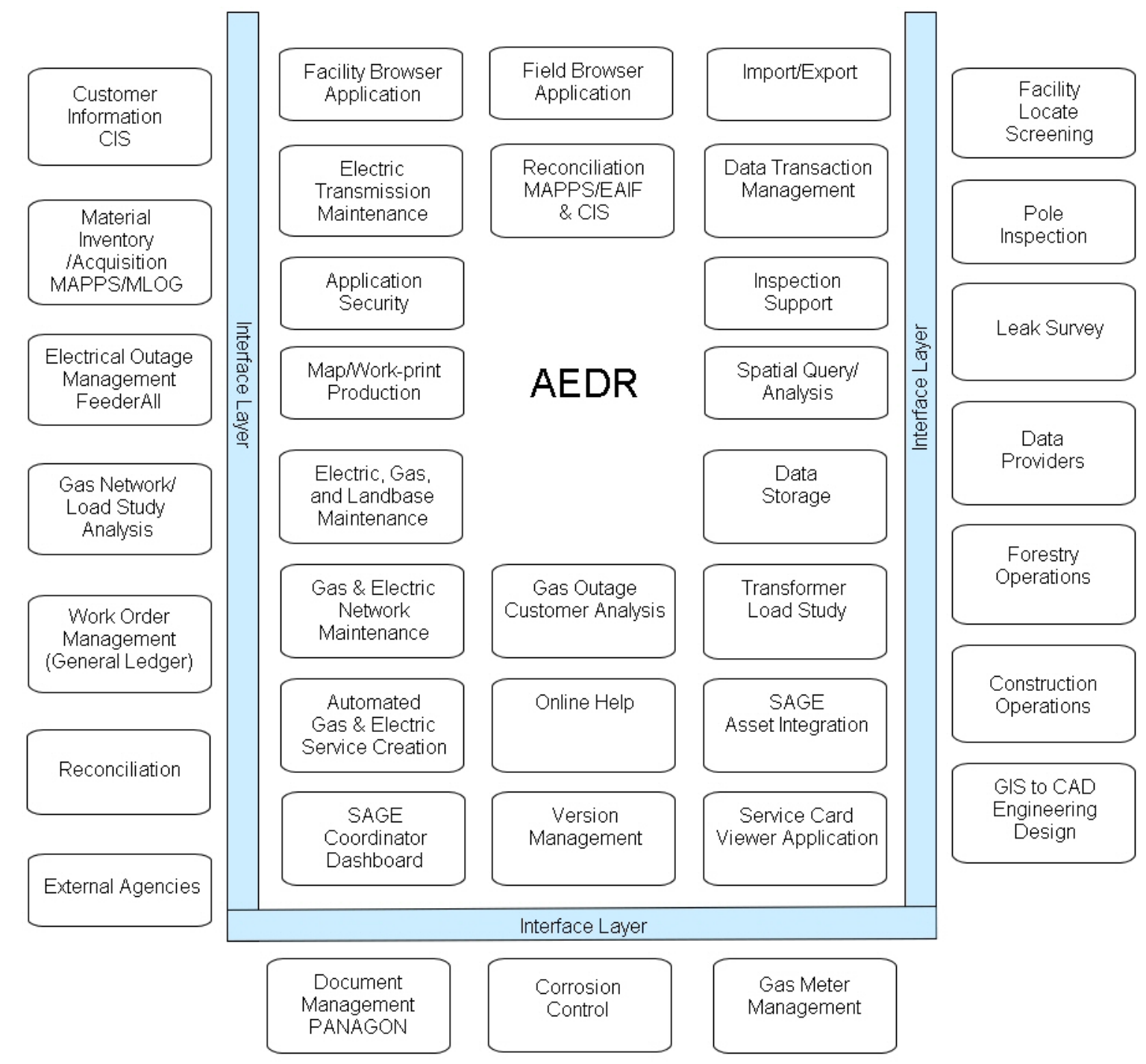

Figure 3-1. NIPSCO GIS final result of the AEDR

Many additional customizations not shown in Figure 3-1 are serving NIPSCO's user community with tools not previously envisioned, but implemented for reasons of interoperability improvement for the AEDR. Business requirements were met and expectations were exceeded. The implementation of the AEDR has had a positive and sometimes dramatic affect on aspects of NIPSCO's data and employees. 
Appendix

Post Implementation Review 


\section{Table of Contents}

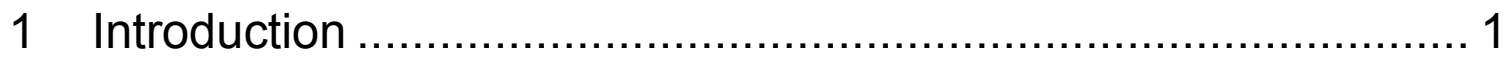

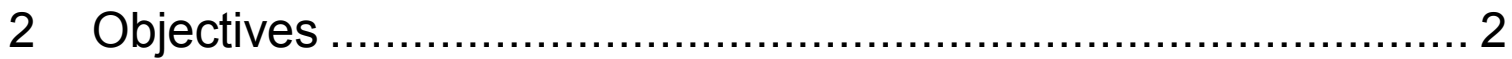

3 Project Implementation Review ......................................... 4

3.1 Project Management .......................................................................... 4

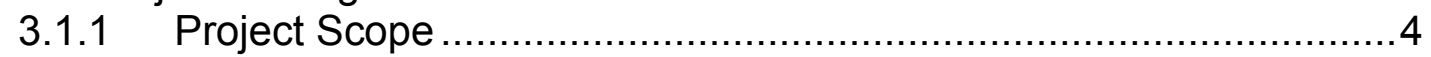

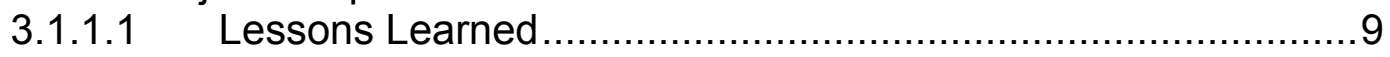

3.1.2 Project Schedule ………………………………………....

3.1.2.1 Lessons Learned ............................................................. 10

3.1.3 Project Resource Management .................................................. 10

3.1.3.1 Lessons Learned ............................................................. 10

3.1.4 Project Methodology ..................................................................11

3.1.4.1 Lessons Learned ................................................................11

3.1.5 Risk Management..................................................................11

3.1.6 Change Control .....................................................................11

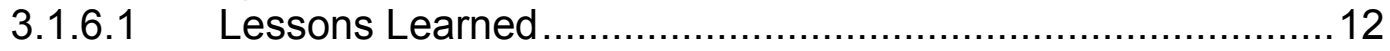

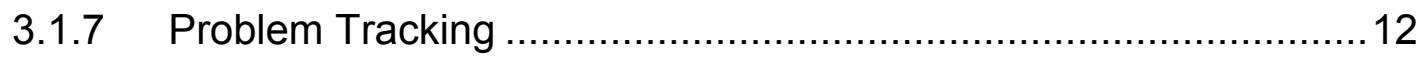

3.1.8 Communications Plan................................................................. 12

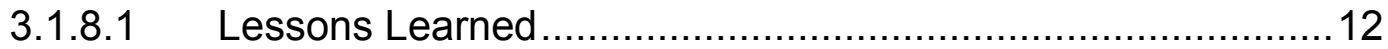

3.2 Functional Requirements................................................................ 13

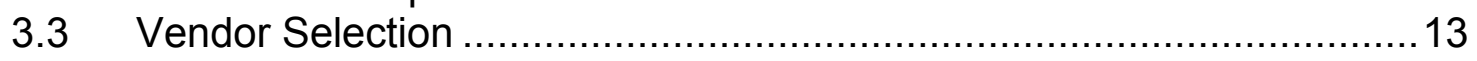

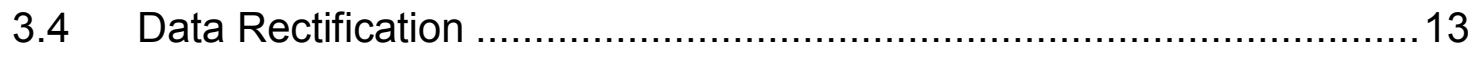

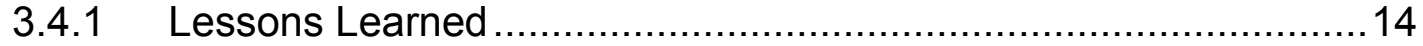

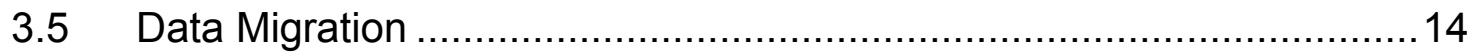

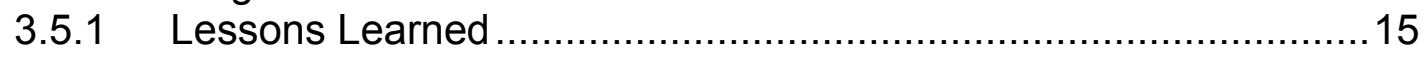

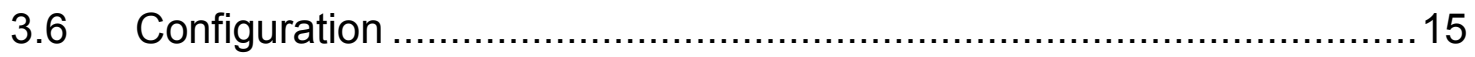

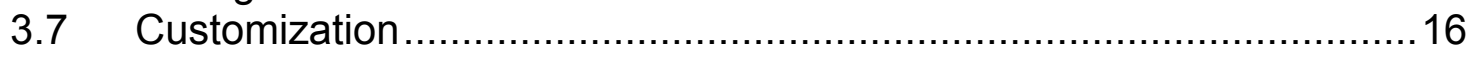

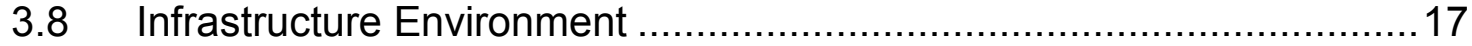

3.8.1 Lessons Learned …………………………………………...... 17

3.9 Interface Development................................................................... 18

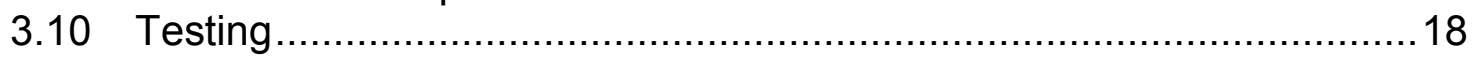

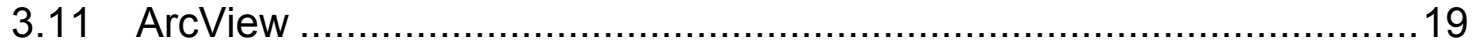

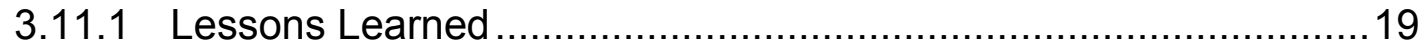

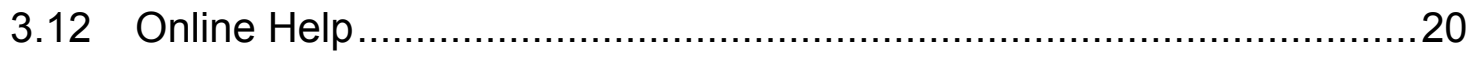

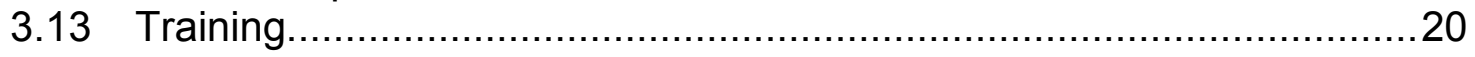

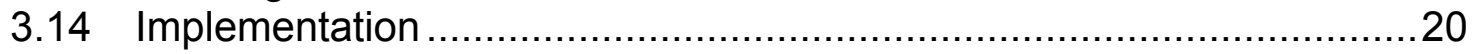

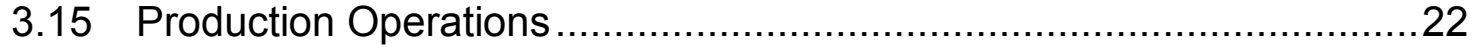

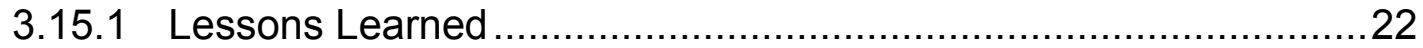

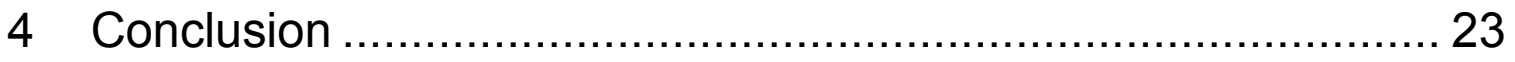




\section{Introduction}

The AEDR is considered complete as defined by NIPSCO terminology, "Used and Useful" or, in other words, an effective and efficient business solution has been successfully implemented and completely transitioned to the business organization.

Although the implementation of the AEDR was highly successful, there are always lessons to be learned during the development and implementation of any large scale and complex project. The purpose of this Post Implementation Review is to report the project successes, the lessons learned and to provide an assessment of whether the business solution meets the original goals of the project. Additionally, usability and performance to efficiently sustain business operations must also be considered.

In reference to the problem and solution domain (Figure 1-1) described in the "Automated Energy Distribution and Reliability System Status Report" http://www.nrel.gov/docs/fy08osti/42265.pdf, this post implementation review is intended to report on the effectiveness of the ultimate solution.

\begin{tabular}{|l|l|}
\hline The problem of & $\begin{array}{l}\text { A limited automated mapping application } \\
\text { that does not provide all of the data all of } \\
\text { the time }\end{array}$ \\
\hline Affects & $\begin{array}{l}\text { Records, Engineering, Corrosion Control, } \\
\text { Gas Meter Management, Gas Systems } \\
\text { Engineering, Locate Screening, Field } \\
\text { Crews, Analysis Engineering and Maps \& } \\
\text { Records }\end{array}$ \\
\hline The impact of which is & $\begin{array}{l}\text { Business decisions made on questionable } \\
\text { or insufficient data and the length of time it } \\
\text { takes to perform key operational activities. }\end{array}$ \\
\hline A successful solution would & $\begin{array}{l}\text { Promote safety, enable sound business } \\
\text { decisions, increase efficiencies, increase } \\
\text { the visibility of data, increase the user base } \\
\text { and compliance with governmental } \\
\text { requirements. }\end{array}$ \\
\hline
\end{tabular}

Figure 1-1. The NIPSCO AEDR problem-solution domain 


\section{Objectives}

In order to achieve the solution described in Figure 1-1, specific objectives were targeted. The objectives included:

- Elimination of redundant data and systems

- Implementation of an automated business rule-driven application to manage GIS data

- Improvement of data quality and integrity

- Gas and electric load study integration

- Establishment of improved and integrated information to satisfy external entities' needs and identify opportunities such as

1. Identification of critical habitats of threatened or endangered species

2. Exploration of alternative energies, such as wind energy, combined heat and power (CHP) units and distributed resources/generation (DR/DG)

3. Impact of future installation of facilities located near the Indiana Dunes National Lakeshore to enable environmentally sound decisions

- Gather and provide information and participate in IEEE standards development work groups.

Figure 1-2 represents the intended database-driven GIS solution managing all of NIPSCO's gas, electric and landbase objects and the interoperability available to the interfacing technologies. 


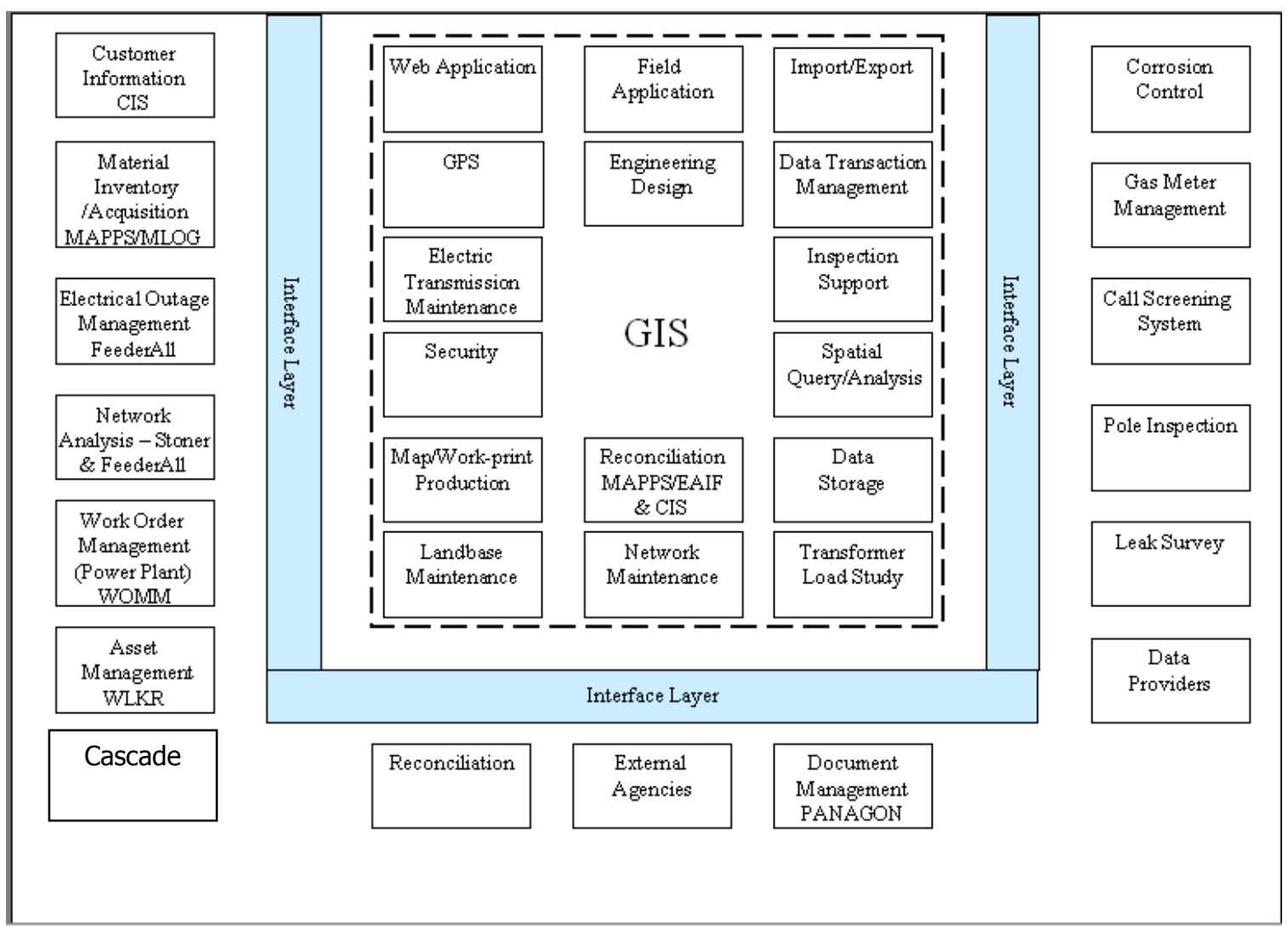

Figure 1-2. NIPSCO GIS approach to AEDR

Section 3.1.1, the project scope, further defines the project deliverables including items to be researched for inclusion in the AEDR. "Research" does not necessarily mean "Implement". Rather, implementation of additional tasks was based on feasibility and budget. Further discussion of these research items are contained within this report. 


\section{Project Implementation Review}

\subsection{Project Management}

\subsubsection{Project Scope}

At a high level and in accordance with NIPSCO's Vision Plan, the scope of the project included the following:

- Replacement of Outfield (an application that runs on top of AutoCAD to facilitate data entry)

- Data migration of both EDFS (Electric Distribution Facility Services) and AutoCAD data to the new GIS and a method for managing electric asset data within the GIS

- Data cleanup, including positional re-adjustment

In addition, these existing interfaces will be maintained or re-written to accommodate the new GIS:

- IRTH - One Call Application

- MAPPS - Material, Accounts Payable and Purchasing

- CIS - Customer Information System

- EAIF - Engineering Accounts Information File

- CADOPS - NIPSCO's Outage Restoration System

- FeederAll - Electric Load Study

- MLOG - Material, Labor and Estimating

- OMS - Gas Outage Management

- Field Browser - Disconnected Field Facility Viewer

- Facility Browser - Web Facility Viewer

- SynerGEE - Gas Load Study

High Level Project Scope Results: The AEDR was implemented in two phases as detailed in the reports: "Automated Energy Distribution and Reliability System Status Report” http://www.nrel.gov/docs/fy080sti/42265.pdf and, “Automated Energy Distribution and Reliability System (AEDR): Validation Integration - Results of Future Architecture Implementation” NREL Report No. SR-581-43432.

All high level in-scope project items outlined in NIPSCO's original Vision Plan were implemented including re-written or replaced interfaces. In addition, several new tools and functionality not in the original scope, but identified as tools that would enhance or assist in the user or management experience were also implemented as the budget \& schedule allowed. Also, the coordinate system was upgraded from Indiana State Plane West NAD 27 to Indiana State Plane West NAD 83. The data cleanup and positional rectification including re-adjustment to the new coordinate system was outsourced to a vendor. 
Some of the more detailed in-scope project items became out-of-scope during system development. Management decided to postpone some of these original in-scope items and make them part of the new enterprise GIS development initiative. These out-ofscope items are detailed in subsequent tasks results.

The DOE/NREL subcontract further defines scope with the following list of tasks:

\section{Task 1 - Selection of GIS software platform}

This task shall establish the GIS software platform that could meet NIPSCO's business requirements and criteria. The initial implementation will entail the Immediate Conceptual Architecture and the Future Conceptual Architecture, both undertaken in this subcontract. The selection of the software platform will accommodate future expansion.

Task 1 Results: The ESRI GIS platform was chosen. ArcGIS/ArcFM best meet NIPSCO's business requirements in comparison to the other vendors researched and ESRI is the dominant industry leader. The entire NiSource organization has chosen to implement the ESRI platform in the future. The AEDR can fully and easily migrate and integrate into the enterprise platform bringing with it all the useful and important customizations created as a sole NIPSCO project.

Task 2 - GIS Project Definition and Demonstration of Integration

This subtask shall address the following activities:

- Implementation of a Software Engineering Process (SEP)

- Inception Phase

- Elaboration Phase

- Progress of sub-tier subcontractor

- Review and evaluation of each SEP component

- Validate subsets of the database

- Interface integration

Task 2 Results: The implementation of the Software Engineering Process (SEP) is addressed in detail in Section 3.1.4 Project Methodology.

Six sub-tier vendor companies were engaged throughout the project. Contractors from the vendor companies were frequently evaluated for their contributions to the project. Non-contributing contractors were replaced with other contractors, some from the same vendor company and some from one of the other five vendor companies.

Task 3 - Immediate Conceptual Architecture Implementation

In summary, this task involves implementing the "Immediate Conceptual Architecture" according to NIPSCO requirements and criteria. This first implementation (Phase I) shall involve the following:

- Implement a database-driven GIS solution that will manage all of NIPSCO's gas, electric, and land-base objects.

- Replace the core GIS maintenance product (Outfield) with the GIS solution, merge the Electric Distribution Facility Services (EDFS) data into the new GIS 
and maintain the existing interfaces to Customer Information System (CIS), Engineering Accounts Information File (EAIF), Material Accounts Payable and Purchasing System (MAPPS), Advantica Gas SynerGEE; ABB CADOPS, Gas Outage Management System (OMS), AutoCAD formats and Infobuilder.

- Implement the Immediate Conceptual Architecture.

- Configure the software.

- Improve the operational efficiency and data accuracy through the elimination of redundant data entry.

- Reduce the operational costs.

- Improve customer satisfaction through improved reliability and customer service.

- Implement a system with flexible configuration capabilities that enables NIPSCO to easily make changes over time.

- Replace the current web-enabled product, Facility Browser, with an integrated product on the ESRI platform.

- Replace the current disconnected field viewer, Field Browser, with an integrated product on the ESRI platform.

Task 3 Results: The ArcSDE solution now manages all of NIPSCO's electric, gas and land based objects. All of the data is contained within one database, thus eliminating the data maintenance redundancy and improving data integrity. Operational efficiencies are gained when a user enters the data into a single database and because of the data integrity improvements, correction activities are minimized.

Outfield was replaced with ArcEditor and the Stand Alone Geodatabase Editor (SAGE) was built to contain and maintain EDFS' tabular data. The EDFS data with a spatial component has now been merged into the GIS. The benefits for this arrangement are cost savings in that they reduce the operational costs for users who only require access to the tabular data contained within SAGE, thus saving the costs of additional (and expensive) ArcGIS/ArcFM licenses.

All interfaces have been revised or replaced with the exception of Infobuilder. It was determined that this interface was no longer required.

Improved reliability has had a dramatic affect on customer service especially with the new tools built to support the Gas Integration Center. These automated tools significantly improve customer satisfaction by improving dispatch response time for customer re-lights following a gas outage. Other departments within the organization are finding this tool useful, among them gas load studies and gas meter management personnel.

Over one hundred fifty customizations developed over the 1.5 years from the first AEDR implementation attest to the flexible capabilities of the software. NIPSCO has a very knowledgeable and responsive GIS project support team capable of providing competent technical support services which also serves the version upgrade process very well. For instance, the upgrade from ArcGIS 8.3 to ArcGIS 9.2 was trouble-free and finished several hours ahead of schedule. 
The legacy Facility Browser application was replaced with ESRI's ArcIMS web-enabled product and the Field Browser application was replaced with ESRI's ArcReader. Both applications served the purpose at that time, and ArcReader continues to remain the right choice for the price (ArcReader is a free viewer), however a newer and more efficient replacement for ArcIMS has emerged and while NIPSCO will remain on the existing ArcIMS platform for the time being, the enterprise GIS is intended to use the new product, ArcGIS Server in the future.

\section{Task 4 - Future Conceptual Architecture Implementation}

In summary, this task involves implementing the "Future Conceptual Architecture." This phase of the implementation (Phase II) shall be based on the future NIPSCO priorities and lessons learned from the first implementation of the architecture.

The following defines additional functionality / enhancements that are to be researched for inclusion in the GIS:

- The GIS is intended to continue to provide an integrated repository for all outside plant records data and the ability to interface to external systems in addition to new systems for related data.

- The GIS is intended to provide workflow transitioning and multiple data views (e.g. existing vs. proposed).

- The GIS is intended to improve reconciliation efficiency by supporting design posting vs. re-digitizing.

- The GIS is intended to continue to improve network model integrity through strict enforcement of network model / data validation rules and will provide an environment for creating additional rules.

- The GIS is intended to provide support for compatible unit design and design estimating.

- The GIS is intended to provide enhanced field functionality to improve data integrity.

- The GIS is intended to provide enhanced leak / repair capability.

- The GIS is intended to provide the additional ability to support digital photos of stations, poles, etc.

Task 4 Results: Additional items requiring research to determine functional feasibility for inclusion in the AEDR were investigated in the Future Conceptual Architecture Implementation. The "existing versus proposed" functionality dictates the implementation of Telvent Miner \& Miner's Designer product. There were many user proponents driving the decision to implement the Designer product, and it was investigated and determined that it would be exceptionally useful not only for engineering, but also for the electric outage management group. However, the decision to develop the Designer application was postponed until a future date when the enterprise GIS is implemented. There are a handful of engineers utilizing the Designer application for its robust engineering analysis tools, however the Designer application has not been implemented in production to support the true engineering design process and therefore NIPSCO, independent of the enterprise GIS will not realize the efficiencies obtained by 
supporting engineering design posting until the enterprise GIS is implemented sometime in the future.

Electric network model integrity and connectivity has dramatically improved through increased validation, quality assurance team tracing activities and reporting tools that simplify review and correction of the geometric network.

Compatible unit design and estimating is supported by maintaining the link to the legacy interface.

Field functionality remained in a view-only state in lieu of adding redlining or another type of field update capability. However, the new ArcReader replacement for VoloView brought not only new ways of viewing the data not possible using legacy VoloView, but also all of the GIS data. Both tabular and spatial data is now available to the field crews who could previously view only spatial data in the legacy system.

Leak Survey and support of digital photos was also postponed until the enterprise GIS is implemented at a future date.

\section{Task 5 - AEDR Studies}

Identify any additional studies that would enhance the usability of the AEDR such as: Conduct a preliminary study to identify distributed generation and the high probability regions where interconnection and integration of DR/DG for example, CHP, wind energy systems, etc. that may provide substantive returns on investment.

Task 5 Results: The wind energy study was most interesting in that using NIPSCO data via AEDR, we were able to work directly with NREL resources and their Renewable Planning Model (RPM) expert to identify geographical areas where potential harvest of wind energy may be feasible. The depth of the study included not only the selection of potential sites, but also the logistical concerns as well as an evaluation of the type of turbine appropriately suited for the site, and finally, the pros and cons for each selection site were presented in the report. This report can be found as part of the "Validation Integration - Results of Future Architecture Implementation" NREL Report No. SR-58143432 .

\section{Task 6 - Post Implementation Review of AEDR}

Under this task, (the purpose of this Post Implementation Report) conduct a post implementation review of NIPSCO's AEDR, and identify any outstanding critical success factors (factors that have not been met or lessons learned) and incorporate revised aspects into the AEDR.

\section{Task 7 - Standards Development and Implementation}

In June 2003, the IEEE Standard 1547 Standard for Interconnecting Distributed

Resources with Electric Power Systems was approved as an IEEE standard. Additionally, other related 1547 series of connection standards were being developed, including the P1547.3 draft guide for monitoring, information exchange, and control of distributed 
resources (DR) interconnected with electric power systems. The goal of this guide was to present a standardized approach to communications with DR devices that allows for a wide range of business activities, reliability, interoperability, security, and selfdescription and automated configuration. Specifically, the P1547.3 draft outline includes sections on user/stakeholder needs, protocols, legacy systems, security, business processes, operation processes, and information models. NIPSCO will be active in the 1547 standard process, attending meetings, gathering information, developing, reviewing and providing feedback on the standard.

The objectives of this task are to provide technical support to help develop and implement the IEEE 1547 series of standards in a timely manner. This includes providing references and background information, writing draft inputs, participating in systematic reviews, and, promoting 1547 implementation. The task activities include participation in 1547 series of standards development meetings and related activities such as teleconference calls, especially for P1547.3, and, educating/informing colleagues, e.g., at the corporate, utility/industry, state, regional, and national level, as well as helping incorporate implementation of the 1547 series of standards.

Task 7 Results: In fulfillment of this task, NIPSCO was honored to be asked to participate in the IEEE Standards Development and Implementation of the P1547.3 standards. In excess of one hundred twenty-five hours of active participation, NIPSCO was able to provide valuable contributions to the process and communicate the benefits of the 1547.3 standards both internally and to external business partners in a timely manner.

\subsubsection{Lessons Learned}

Scope changes evolved throughout the project and were either approved or denied. Although we successfully implemented the original scope of functionality and much more, some good opportunities to increase productivity and information accuracy were lost when changes that were recommended by the project team were denied or postponed. These changes could have been implemented earlier and easier at NIPSCO where users would already be trained and then migrate/integrate the functionality either along with or as a phase two implementation of the enterprise GIS.

\subsubsection{Project Schedule}

Management set the first phase delivery to be completed by the second quarter 2005 . We had to make changes to our project plan timeline to more accurately reflect a realistic timeframe. After a thorough estimation of the work requested, it was determined that the first or second quarter of 2006 was more likely achievable. This proved true once problems with the rectification and migration processes were encountered.

The project came in at the very end of the expected timeframe mainly due to the rectification and migration process problems. Contingency to account for these types of problems was originally built in to the schedule during the estimation phase, however management was motivated to implement as soon as possible making the target dates very aggressive. Many of the project team members were close to meeting their 
deadlines, however as nearly all projects encounter, there were resource issues that prevented some deadlines from being met in a timely manner.

\subsubsection{Lessons Learned}

Data migration and data rectification were, as usual, on the critical path and problems with both migration delivery quality and team leader inexperience in this area caused delays for the entire project. A team leader resource experienced in data migration and data rectification should have been assigned to manage this process if both could have been made available.

\subsubsection{Project Resource Management}

A variety of vendor resources from six different companies brought not only extensive skills to the table but also the competitive nature that comes with rival companies working together on the same project. Effective vendor management was key in preventing problems. Rules were set and vendors not abiding by the rules were removed until we were left with a set of vendors that worked well together and adequately supported their NIPSCO counterparts. NIPSCO resources brought a wealth of knowledge to the project, however they too were not without issue. Reporting structures made it difficult to ensure project commitments were kept.

Team leads were assigned to each component of the project. They were chosen based on best skill level for a particular component, however for some components we simply had no experienced resource available. These components were monitored to the extent possible.

Although users were highly engaged during the functional requirements phase, the users (subject matter experts) outside of the project team were not formally assigned to the project, and GIS project team members were asked to fill the gap in specific areas even if they were only marginally knowledgeable of the subject matter.

\subsubsection{Lessons Learned}

Managing multiple resources from six competing vendor companies and the NIPSCO project staff was a project management challenge. The talent brought to the project was outstanding, however the workload of managing the mix of vendors both administratively and functionally was much more than anticipated. If the talent is available from a smaller concentration of companies, it would be somewhat easier to manage administratively and possibly less contentious. An internal reporting structure should lend itself to accountability for project work assignments.

Projects are traditionally provided with an insufficient number of resources and/or resources with an insufficient skill set. This project did not break from tradition, and although the project was successfully completed, it did not come easy for many project team members. Every effort should be made to assign a sufficient number of skilled resources directly to the project. 
Lack of functional user involvement committed to the project on a regular basis contributed to pulling project team resources out of their respective areas of responsibility to fill the void. Consequently, some rework was required to support the business case functionality and meeting target dates to stay on schedule became challenging.

\subsubsection{Project Methodology}

Risk mitigation is a considerable factor in ensuring the success of a project. That being said, the identification of high risk and architecturally significant items, and the proof of concept of those items were to be investigated at the beginning of the project rather than at the end. To assist in that theory, an iterative approach rather than the traditional waterfall process was implemented. This type of methodology was followed for several months and subsequently abandoned in favor of a traditional waterfall methodology.

\subsubsection{Lessons Learned}

The iterative approach may work well for small projects, however we found it expensive in both time and cost to use an iterative approach in the development of the AEDR. The methodology was difficult to implement and maintain and the project team found it counter to the intuitive waterfall approach. We concluded that the AEDR was too large and complex for the iterative methodology approach.

\subsubsection{Risk Management}

Risk management was one of our strengths. Risks were defined in terms of "technical" and "general". A spreadsheet was developed containing a comprehensive list of the different types of risks under each category and team leaders were assigned responsibility for a number of different risks. Risks were weighted and assigned a severity. Meetings were held on a weekly basis and action items assigned until the risk was mitigated or substantially minimized. Risks identified throughout the duration of the project were added to the spreadsheet, assigned and managed appropriately. Appropriate management of risk contributed to the overall success of the project.

\subsubsection{Change Control}

Project change control procedures were written, communicated and stored in the project library. Changes to scope, the data model or other enhancements required a change control form containing detailed information and required approval for implementation. While this worked well for GIS project team application changes, infrastructure (server) changes occasionally caused problems, including shortly before production implementation when critical servers were taken out of commission for several days without warning. In the production environment, lack of infrastructure change control occasionally results in Monday morning AEDR unavailability due to unknown infrastructure modifications. After numerous unsuccessful attempts to insert the GIS project team into the infrastructure change process, we wrote our own infrastructure change control process and presented it to the Information Technology group. This change control process is under IT review at the time of this writing.

With the exception of infrastructure, the formal application change control process served the project application development process well. The application change control process 
changed slightly in the production environment. Every change to the production environment no matter how small, i.e. symbology, configuration etc. is documented, approved and stored in the project library for future reference, maintenance and verification of approval.

\subsubsection{Lessons Learned}

AEDR application change control is easy to manage with the current process in place, however infrastructure (server) change control continues to be challenging and timeconsuming as all the bugs are not yet worked out of this process. Infrastructure change management should be clearly defined prior to production implementation. However, this type of process is governed by the Information Technology group and if that process cannot be controlled then even the best application suffers from unnecessary downtime and this promotes loss of productivity and lack of user buy-in.

\subsubsection{Problem Tracking}

Implementation of a third-party problem tracking program was one of the best solutions toward minimizing the efforts and support of a home-grown system. NIPSCO continues to lease a highly configurable third party solution in the production environment.

Problems were rated critical, high, medium and low with the focus on resolving the critical, high and many of the medium problems, and then planning strategy for resolving remaining problems after production implementation.

On a daily basis, new problems were reviewed, priorities were adjusted if necessary and resources assigned to resolve. Problems were tracked via the project plan until it became too unwieldy, and then they were tracked via reporting from the tool. Problems requiring project team discussion and / or assistance were added to the daily / weekly project status meeting agenda. Problems that were not being resolved were escalated, sometimes to management to resolve. Problem tracking continues in the production environment in a similar manner with a "Help Desk" support function added to the process.

\subsubsection{Communications Plan}

NIPSCO created an organizational change management communication plan but did not follow the plan on a regular basis, mostly due to lack of resources.

Project plan reviews were scheduled weekly, and at times, daily when there was a great deal of different activities occurring. Still, project team members felt they did not receive sufficient communication regard project activities. The project plan was the mechanism to ensure that all project team members were aware of the overall project status as well as the tasks occurring in each component. Regular project plan review aided the project team in identifying and solving dependencies between interrelated tasks.

\subsubsection{Lessons Learned}

Infrequent organizational change management communication resulted in users and potential users not being informed of project progress in a timely manner and the benefits of the AEDR were not widely realized until it was in production for nearly a year. A 
resource from Corporate Communications assigned to the project team would have helped ensure that the project received the publicity early on and throughout the project.

Although the project plan was reviewed at times on a daily basis, some project team members wanted more involvement with the entire project. This was simply not possible due to the small number of resources trying to meet specific target dates. Each resource was set up in team fashion to focus on their area of responsibility. Setting project team members expectations at the outset of the project by explaining that it would not be possible for them to be involved in all aspects of the project may have helped some project team members understand that the enormity of the undertaking, the project implementation timeline, and a thin complement of project team resources prevented everyone from being involved in every component of the project.

\subsection{Functional Requirements}

Functional analysis began with a broad look at the project goals and then refinement of each area within the project with additional detail, functional requirements and software requirements that evolved into a functional software design. As the process surrounding each area of the project became more detailed, it was tied back to the higher level components to ensure consistency, scope, and coverage. Users were highly involved in defining the functional requirements for the AEDR through a series of sessions where like or interfacing current business functions were identified. Functionality identified as in-scope was implemented in the AEDR.

\subsection{Vendor Selection}

The project team spent a significant number of hours:

- refining user requirements

- building an RFP to describe the requirements

- designing a demonstration script to allow the project team to accurately and fairly compare each vendor's product offering

- attending and participating in vendor software demonstrations, and

- finally, ranking and scoring each vendor against the evaluation via a pairwise comparison.

Their diligence to this effort paid off in that ESRI not only ranked highest in the pairwise comparisons, and provided the highest value per dollar over a 5 year period, but also is now currently the world leader in the GIS software industry. The vendor selection process was organized, methodical, comprehensive and objective. Consequently, the GIS project team did very well in the selection of a software vendor.

\subsection{Data Rectification}

In keeping with the objective of improving data accuracy, NIPSCO hired a vendor to rectify the positional inaccuracies in NIPSCO's data and apply a new coordinate system. The size of this effort made it impossible to perform with the current staff. A set of rectification rules for land, gas and electric were designed with the vendor to ensure vendor consistency in the positional adjustments as well as NIPSCO consistency in 
quality checking. NIPSCO was charged with quality assurance checking fifty percent of the delivered data. The vendor was held to delivering the rectified data to ninety-eight percent accuracy.

The coordinate system was updated to prepare the data for rectification. The reprojection from Indiana State Plane West NAD 1927 to Indiana State Plane West NAD 1983 was accomplished within two weeks. Web-enabled data was frozen for the two weeks during the process that was performed over two consecutive weekends. The vendor then rectified all of NIPSCO's data to the new coordinate system.

Using a small subset of NIPSCO's data, a pilot data rectification was conducted to ensure that the vendor could perform the work in a manner acceptable to NIPSCO, refine both vendor and NIPSCO quality assurance processes and to better estimate the amount of time that would be necessary to complete the entire project. Data was sent off to the vendor in manageable batches (AutoCAD tiles) of geographical areas. Pilot data was redelivered to the vendor twice, so it took the vendor a total of three deliveries to reach the $98 \%$ accuracy threshold with a limited amount of data. Throughout the remainder of the process, up to six deliveries of data were required for the vendor to reach the $98 \%$ accuracy requirement.

\subsubsection{Lessons Learned}

The process of piloting the effort did not provide the accurate information anticipated. We expected that the data quality would improve as the vendor became more familiar with the data and rectification rules. Those expectations were not met. Instead of data quality improving, it degraded requiring a second delivery for most of the batches and up to six deliveries of data for some batches. In the end, fifty-nine percent of all features were quality assurance checked and the vendor did meet the ninety-eight percent accuracy for those features checked. It is unknown whether the ninety-eight percent accuracy was met for the forty-one percent of features for which quality assurance was not performed. It should be noted that NIPSCO quality assurance checked the most difficult and problematic features first.

The rectification process and the data migration process testing were performed by the same vendor simultaneously. A NIPSCO team leader was assigned to manage and oversee both operations. Shortly into the processes, the NIPSCO team leader was replaced by another team leader. It is recommended that if at all possible, a single resource should not be assigned the responsibility for both rectification and data migration for a database the size of NIPSCO's regardless if the migration / rectification vendor is one in the same.

\subsection{Data Migration}

NIPSCO's data was stored in three main sources: AutoCAD drawing files, Microsoft Access database and a mainframe DB2 database. The vendor was required to segment, aggregate, associate, and / or manipulate the data in order to create appropriately segmented, joined, related, connected, and populated GIS features and objects. 
As in the rectification process, migration specifications and rules were developed for the vendor, and a pilot migration was conducted to test the specifications, rules and quality assurance processes. Four "test" data migration deliveries were originally planned, with each delivery providing an increasing level of data, and finally a full set of data with the fourth delivery. The fifth migration delivery was intended to be the final migration delivery which would be used in the production environment. Each delivery was quality assurance checked by NIPSCO. NIPSCO used a combination of automated and interactive quality assurance checks to verify the data was migrated accurately and completely.

Due to quality problems with the deliveries, an additional delivery was added.

The final production data migration process spanned six weeks $24 \times 7$ from start to finish and included vendor migration of data, NIPSCO migration of data, interactive and automated quality assurance processes, preparation processes such as building the geometric network and running synchronization processes until finally the AEDR was implemented into production.

\subsubsection{Lessons Learned}

Both rectification and data migration processes were performed by the same vendor simultaneously. A NIPSCO team leader was assigned to manage and oversee both operations. The team lead position was replaced a total of three times throughout the migration effort. Finally the project manager assumed the task. Resource issues were responsible for many of the migration process issues. An experienced and dedicated team leader for the migration process is key to a successful migration. A team leader should ensure that all processes are in place before the first migration delivery is received, and should monitor closely and take swift corrective action for unacceptable vendor deliveries.

Data migration was very difficult from both the NIPSCO and vendor perspectives. Prior to the last migration delivery, it was determined that, the segmenting, aggregating, associating, and/or manipulation of the data in order to create appropriately segmented, joined, related, connected, and populated GIS features and objects along with some other migration activities proved too challenging for the migration vendor. In order to complete the migration and implement the AEDR, NIPSCO internal resources wrote in excess of forty migration routines to compensate where the migration vendor was unable to migrate the data accurately.

Although the data migration process was probably the most challenging component of the entire implementation, most of the NIPSCO GIS team worked hard on the last two deliveries to ensure its success.

\subsection{Configuration}

Base configuration constituting end user preferences was captured during a series of interview sessions with key end users. The configuration was entered directly into the ArcFM Properties Manager during the interview sessions. This was determined to be the 
best approach to capture and retain this information which was exported and saved in standard XML files.

Once the base configuration was complete, the configuration for all custom software components was also applied to the geodatabase via the ArcFM Properties. Each of the custom installations included an Operations Guide which details the custom configuration for both the Model Names and the component assignment. These were applied in the same manner as the base configuration.

This approach ensured configuration completion in a timely manner.

\subsection{Customization}

NIPSCO created over one hundred fifty customizations. Some of the most notable and valuable are:

- SAGE - Electric asset management integration with AEDR.

- Interactive Clerk Performance Analysis - Allows an administrator user to run interactive analysis on the performance of an individual clerk.

- Structure Span Lengths Query - Allows all users to query the structure span lengths by querying on any structure ID.

- Coordinator Dashboard - Provides a tool to view and manage electric asset information.

- Field Browser Circuit Isolation - Allows the user to see the extent and control points of each individual circuit for a quick analysis of scope, location and isolation devices.

- Service Card Viewer - Provides the ability to view service information based on customer information.

- Online Help - Provides detailed help customized to NIPSCO's processes.

- Custom Network Adapter - customized to export a custom view of the AEDR electric networks.

- All Edits Report - Provides a detailed report of all new records, deletes, and updates in a session.

- Non-locking Reconcile - Allows multiple quality assurance users to simultaneously perform a "non-locking" reconcile against SDE.Default.

- CIS Service Request Automation - Allows clerks to import customer data into the GIS creating a physical location for the customer installed service. This tool also supports updating and transferring the service to an engineer for design and finally the transfer back to the clerk as the as-built update.

- Session Manager Approve Session for Batch Reconcile and Post (BRP) Subtask Marks a session as approved and updates the session state to "Pending Post". which triggers the BRP process to automatically reconcile and post the session

- Session Manager Auto Submit Session For Post Subtask - Subtask to automatically approve and submit to the BRP queue (pending post state).

- Gas Customer analysis - Allows a gas operations technician to select a set of gas mains that are included in an outage and automatically create buffers around the selected pipes which then allows them to locate customers within the buffer. The 
technician can then export a master list of the customers into MS Excel and create the actual field Gas Outage / Re-light forms in MS Excel all with the click of a few buttons. Customers benefit from faster re-lights due to better allocation of resources during an outage.

The many customizations were the result of user requests which make this system truly user-friendly and add to the depth of value the AEDR brings to NIPSCO.

A recent system upgrade to ESRI 9.2 was performed in 1.5 days, successfully, over a weekend, and came in well ahead of schedule.

A useful GIS is one that fulfills the business requirements and creates a user-friendly environment. An organization should not be concerned with "too many customizations to manage". Rather, the productivity gains and ease of use should dictate customization. We have found that as long as the upgrades are carefully planned, the testing is thorough (it will take longer depending on the number of customizations) and documentation is in place, upgrades should be easy and successful with minimal if any disruption to the user.

\subsection{Infrastructure Environment}

AEDR runs in a four Windows 2003 server farm Citrix environment. The database is SQL Server running on a Windows 2003 server.

We have found the Citrix implementation has a number of advantages:

- Application and data access are moved to the computer room in proximity thus reducing network bandwidth requirements

- Relieves the need to regularly upgrade client workstations to keep up with increasing software system demands

- Administration is centralized resulting in a reduction in administration costs

- Ability to rapidly deploy applications, including web-based deployment options

- Provides a secure computing environment since all data can be protected in the server room while only displays are sent to the client device.

While this report only attempts to address the infrastructure at a high level, many other infrastructure concerns beyond the control of the project team have hampered the ability of the GIS to perform optimally. These concerns are related to an aging infrastructure, bandwidth issues and aging servers.

\subsubsection{Lessons Learned}

Performance has become an issue since the server hardware and software has not been upgraded in a timely manner, and the increasing popularity of the AEDR has added strain to the antiquated infrastructure resulting in slower performance and user dissatisfaction. Our Information Technology department is currently working on a solution to ease but not completely resolve the problem. Keeping up with server hardware and software improvements is a critical success factor. In general, NIPSCO is satisfied with the Citrix/SQL Server configuration, but not the aging infrastructure. 


\subsection{Interface Development}

All of the required interfaces defined below have been implemented and are being used in production today with the exception of FeederAll. Although the interface has been developed, the end user is not prepared to accept the interface at the time of this writing.

- Facility Browser - Web Facility Viewer

- SynerGEE - Gas Load Study

- MLOG - Material, Labor and Estimating

- OMS - Gas Outage Management

- IRTH - One Call Application

- MAPPS - Material, Accounts Payable and Purchasing

- CIS - Customer Information System

- EAIF - Engineering Accounts Information File

- CADOPS - NIPSCO's Outage Restoration System

- FeederAll - Electric Load Study

- Field Browser - Disconnected Field Facility Viewer

AEDR Facility Browser (web viewer) was not implemented until six months postproduction due to resource issues.

After much investigation, it was determined that vendor modification of SynerGEE's DataPrep to conform to the ESRI platform was cost prohibitive, therefore NIPSCO wrote their own data extraction tool at a significant cost savings and a gain in functionality.

MLOG (Material \& Labor Estimating) was originally scheduled to be replaced with another product. A fair amount of effort went into the interface development, however in the end, the product was abandoned and the original MLOG application interface was maintained. Since we were only responsible for the interface, we simply re-directed the AEDR to the legacy MLOG interface.

During development, scope was changed in that the OMS (Gas Outage Management) interface was removed. Since the user requirements were critical, a customization, the Gas Customer Analysis tool, was implemented in place of a formal and fully developed Gas Outage Management system. The Gas Customer Analysis provides the end-user with a critical tool for restoring customers after a gas outage.

The remaining interfaces were all built and implemented on schedule and to end-user satisfaction.

\subsection{Testing}

Formal test cases were created for every component and testing was executed within a controlled test system environment. Problems resulting from testing were either fixed and re-tested on the fly or were logged as a problem and assigned a severity for future correction. 
All customizations, components, etc. follow the same testing procedures of unit testing, system testing and user acceptance testing using test cases specifically developed for the component. Test cases that fail during the final test phase (user acceptance testing), are corrected and re-tested before promoting into production. A one hundred percent passing of test cases is required prior to production implementation.

An additional (bonus) level of testing was performed by using test scripts that were written to conduct stress testing of the application to determine whether the system performed adequately under a full user load. The performance/stress testing was conducted shortly before the AEDR was implemented into production.

Testing of the AEDR prior to production was extensive and thorough. Issues were reported through a problem tracking system, prioritized and corrected prior to production implementation. The proof of an adequately tested application is reflected in the production rollout. The AEDR went into production with only a few minor problems that occurred over the first several weeks and those problems were easily and quickly addressed. No user downtime was experienced. The testing approach followed industry standards and worked well for this implementation. Performance testing with a full complement of users simulating an editing environment provided assurances that the application would perform adequately in the production environment.

\subsection{ArcView}

AEDR ArcView was originally intended to be a minimally used application. Shortly after the AEDR went into production and users began to realize its potential, use of AEDR ArcView became one of the most desirable user GIS tools. Originally, a limited number of users were expected to utilize ArcView: Executive, Central Operations Supervisor, Field Manager, Corrosion Personnel, Facility Locate Screening and Construction Maintenance and Service. Additionally, eighty engineers, six Electric Distribution Systems Resource Management employees, forty Gas Operations Integration employees, six Rate Department employees, twenty-two service engineering technicians and an assortment of a smaller number of users have been granted access to ArcView, bringing the total number of users from an estimated twenty-five to one hundred sixty.

\subsubsection{Lessons Learned}

Although we had adequate concurrent licenses to support the users, and were able to train and support new users from a GIS project team perspective, we did not anticipate the popularity of the AEDR would grow as quickly as it did. Nor did we anticipate the traditional server refresh activities would be postponed from the standard three year refresh. Our server hardware and software are six years old as of this writing. Since the server hardware and software had not been upgraded in a timely manner, and as the user base continues to grows, performance is increasingly impacted. As stated in the previous "lessons learned" in the Infrastructure Environment section, keeping up with server hardware and software improvements is a critical success factor. Future implementations would be well served to ensure that the infrastructure is guaranteed to remain on a pre-set upgrade track. Communication should be conducted such that management clearly understands the consequences of an aging infrastructure on a GIS. 


\subsection{Online Help}

The ESRI online help provides an abundance of assistance for the common user. However, as NIPSCO implemented autoupdaters, added functionality and interfaces and even basic GIS operations, we intended to ensure that a user-friendly, comprehensive web-based "user manual" that described how to operate the GIS and interact with custom interfaces would be available within the AEDR. Common GIS operations were included specific to each commodity such that all operational assistance for all AEDR components could be provided in one centralized AEDR Online Help.

One centralized Online Help system eases updating and distribution to a large number of users. Changes are required to be updated in one location rather than distributing manual modifications to the user community. This ensures that all users are accessing the current version of the operating instructions via the Online Help. The GIS team simply adds instructions to the Online Help and communicates the change to the users as new functionality is introduced.

The decision to add the custom Online Help tool has realized many benefits including a quick and easy way to provide all updates to all users in a timely and comprehensive manner and made AEDR support less burdensome on a minimally staffed GIS project support team.

\subsection{Training}

The task of training was carefully considered after many different vendors shared concerns that the learning curve would be high with users unfamiliar to GIS. The training effort of planning, preparing and organizing began eight months in advance of onsite training.

Product vendor experts were hired and a training needs assessment was conducted. The GIS team was trained prior to the AEDR development phase and then again "as users" to serve as experimental students in order to refine the curriculum. The experiment was two-fold in that it brought out the training flaws which were corrected prior to user training and also prepared the GIS team to participate as trainers in the "Train the Trainer" program.

Concerned that the users would experience a great deal of difficulty after only one week of training, mandatory practice sessions were set up immediately following training for the subsequent six weeks at which time the AEDR was implemented into production. The "just-in-time" training, requiring the users to spend four hours per day practicing real life business cases in the test AEDR system and providing onsite assistance proved to be the key to ensuring users were accepting and prepared to operate the AEDR in production.

\subsection{Implementation}

The AEDR implementation required a lengthy data freeze. The initial migration vendor estimate to migrate NIPSCO's data was six to eight weeks from the time of NIPSCO's data delivery to the return of the migrated data to NIPSCO. With data preparation on the 
back end of the delivery estimated at three and a half weeks, the total data freeze was initially estimated at nine and a half to eleven and a half weeks. The migration of data and the processing and preparation for production usage was continuously refined until the project team compressed the total data freeze (including vendor migration) down to seven weeks. While management was not in favor of building a seven week backlog of work, they did understand the benefits of having a state-of-the-art GIS would far outweigh the inconveniences of a short-term overload of work.

The Implementation Plan/Schedule governed the production implementation of the AEDR. Over the course of two months preceding the implementation, preparations were underway and the Implementation Plan was constantly being refined. Each of the following tasks was identified and assigned a timeframe:

- configuration and application testing

- Citrix software installs

- performance testing

- GIS team training

- user training environment setup, and

- preparation of data for delivery to the migration vendor.

Once the data was delivered to the vendor, record clerk training and final integration testing was performed. The vendor migration process spanned sixteen consecutive days and when the data was returned to NIPSCO, it took several days to load the data into the migration instances to prepare it for both automated and interactive quality assurance checking and simultaneously prepare a separate migration build instance for the remaining migration of data that the NIPSCO team was required to perform.

Over the next three weeks, the data was programmatically and interactively quality assurance checked, data cleanup scripts were run, the geodatabase was configured, ArcFM's FeederManager was run and the separate migration build (NIPSCO's GIS team migration) was quality assurance checked. For two days following the completion of the quality assurance checking process, bulk loads and miscellaneous batch processes were run on the data to complete the preparation for production. Subsequently, we opened up production for a small group of users to ensure that any major problems could be addressed before the entire user base began using AEDR in production. The small group used the AEDR for two consecutive days and when no problems were found, the full production environment was opened to the entire user base. Over the next three weeks, GIS project team members were assigned a production support stand-by role to assist in any major system problems. However, no major problems surfaced and the team mostly addressed individual user training issues.

We attribute the successful implementation process to diligent planning, careful execution and project resources remaining on-track with tight deadlines during the implementation phase. 


\subsection{Production Operations}

Now that the AEDR has been in production for nearly 24 months, the lean GIS project team is increasingly challenged with responding to new requests in a timely manner and also addressing aging infrastructure problems that are not within their span of control.

While the AEDR is a state-of-the-art GIS application, the platform on which it resides is highly problematic. The current configuration of support relies on the GIS team to support the application, and the Information Technology (IT) group to support the infrastructure. The infrastructure includes both server and network components. Additionally, the Information Technology group supports application and database access to the AEDR.

The AEDR went into production during a tumultuous event in the organizational structure. The Information Technology group was currently being outsourced and IT processes were being abandoned and/or rewritten. At the time of this report, steady-state processing remains immature which contributes to lengthy problem solving and slow service response.

Since Information Technology support is outsourced to a vendor who provides many resources overseas, solving application and database access problems has become a timeconsuming and difficult process.

\subsubsection{Lessons Learned}

The lesson to be learned is to try to avoid future large complex system implementations during a time of organizational change. If implementation is required at that time, lowering the GIS project team and management's expectations for support may result in a less contentious situation. 


\section{Conclusion}

Since the purpose of this Post Implementation Report is, in part to provide an assessment of whether the business solution meets the original goals of the project, we need to review the NIPSCO AEDR problem-solution domain shown in Figure 1-1.

The problem of a limited automated mapping application (AutoCAD) that did not provide all of the data all of the time was solved by implementing a state-of-the-art AEDR application which not only manages NIPSCO's mapping data, but also tabular and asset data in one location.

The AEDR has had a positive affect on not only the departments intended to benefit from this implementation, but also Gas Load Studies, Rate Department, Gas Integration Center, Electric Distribution Systems Resource Management, Corrosion Control, Forestry, Operations and other back office users. The AEDR is also having a positive affect on the enterprise GIS development project in that customizations developed specifically for the AEDR are intended to be brought forward in the new application. This leads to a substantial cost savings as the "wheel does not require re-invention".

The "impact of business decisions made on questionable or insufficient data and the length of time it takes to perform key operational activities" has been mitigated through the migration of all of the AutoCAD and all of the asset data into one database in order to eliminate the data redundancy and provide improved data integrity which promotes the ability to make sound business decisions using a single consolidated tool.

The "successful solution would promote safety, enable sound business decisions, increase efficiencies, increase the visibility of data, increase the user base and compliance with governmental requirements" were all solved by the implementation of the AEDR. Some examples of these business solutions are:

- Efficiencies were gained by eliminating the maintenance of redundant data contained in multiple databases and supported by multiple applications, and also efficient project team support of a client application spread across northwest Indiana by distributing the AEDR through the Citrix platform.

- The visibility of the data was increased through the use of the new ArcReader viewer installed on the Field Browser laptops which contains tabular data not viewable in the previous VoloViewer application.

- This also led to the increase in safety by the installation of the circuit isolation component on the Field Browser and improved data integrity from validation routines, data rectification and data cleanup efforts.

- The user base has significantly increased as Transmission and Distribution Engineering, the Integration Center, Electric Distribution Systems Resource Management Department, Corrosion Control, Rate Department and many others have benefited from training and use of the ArcView tool.

- Gathering information to support governmental requirements such as NIPSCO's rate case and the National Pipeline Mapping Submission were made easier by the implementation of the GIS. 
The remaining objectives of "implementation of an automated business rule-driven application to manage GIS data" were accomplished by:

- The implementation of data validation, rules and routines, autoupdaters and domains and led to the improvement of data quality and integrity.

- Gas and electric load study integration was completed and installed into production.

- AEDR easily lends itself to the mapping of critical habitats and future installation of facilities near environmentally sensitive areas.

- A wind energy study was completed in partnership with NREL.

- NIPSCO provided support for the development of the IEEE standards.

In summation, the implementation of the AEDR is considered successful for the following reasons:

- The AEDR is user-friendly (with adequate training) and is able to efficiently sustain business operations.

- Critical success factors were met. (Business problems were adequately solved)

- The project met its stated objectives.

- Effective project management practices were able to support the initiation, development, risk mitigation, testing and implementation of the project.

- The end-users are satisfied with the functionality of the application. 


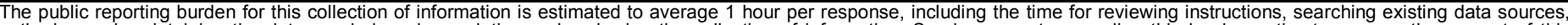

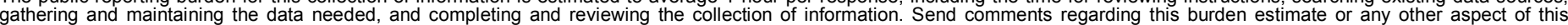

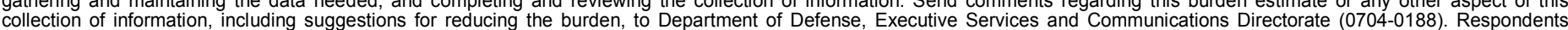

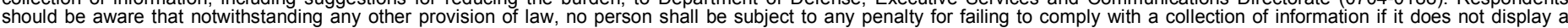

should be aware that notwithstanding

PLEASE DO NOT RETURN YOUR FORM TO THE ABOVE ORGANIZATION.

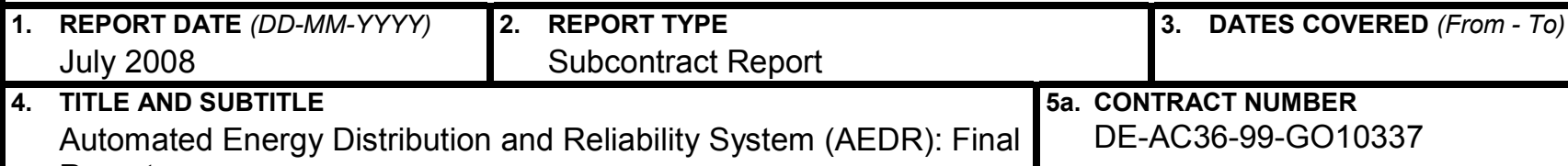

Report

5b. GRANT NUMBER

5c. PROGRAM ELEMENT NUMBER

6. AUTHOR(S)

D.L. Buche

5d. PROJECT NUMBER

NREL/SR-581-43674

5e. TASK NUMBER

DP08.1001

5f. WORK UNIT NUMBER
7. PERFORMING ORGANIZATION NAME(S) AND ADDRESS(ES)

D.L. Buche

NIPSCO GIS

20 South Joseph St.

Crown Point, IN 46307

9. SPONSORING/MONITORING AGENCY NAME(S) AND ADDRESS(ES)

National Renewable Energy Laboratory

1617 Cole Blvd.

Golden, CO 80401-3393
8. PERFORMING ORGANIZATION REPORT NUMBER

RAX-4-33651-01

12. DISTRIBUTION AVAILABILITY STATEMENT

National Technical Information Service

U.S. Department of Commerce

5285 Port Royal Road

Springfield, VA 22161

13. SUPPLEMENTARY NOTES

NREL Technical Monitor: Thomas Basso

14. ABSTRACT (Maximum 200 Words)

This report describes Northern Indiana Public Service Co. project efforts to develop an automated energy distribution and reliability system. The purpose of this project was to implement a database-driven GIS solution that would manage all of the company's gas, electric, and landbase objects. Other NREL reports provide more details: "Automated Energy Distribution and Reliability System Status Report" http://www.nrel.gov/docs/fy08osti/42265.pdf and, "Automated Energy Distribution and Reliability System (AEDR): Validation Integration - Results of Future Architecture Implementation" NREL Report No. SR-581-43432, that includes the NIPSCO-NREL "Wind Generation Integration Study" (June 2007).

15. SUBJECT TERMS

automated energy distribuiton and reliability system; AEDR; geographic information system; GIS; Northern Indiana Public Service Co.; NIPSCO; National Renewable Energy Laboratory; NREL

\begin{tabular}{|c|c|c|c|c|}
\hline \multicolumn{3}{|c|}{ 16. SECURITY CLASSIFICATION OF: } & \multirow{2}{*}{$\begin{array}{l}\text { 17. LIMITATION } \\
\text { OF ABSTRACT } \\
\text { UL }\end{array}$} & \multirow{2}{*}{$\begin{array}{ll}\text { 18. NUMBER } \\
\text { OF PAGES }\end{array}$} \\
\hline $\begin{array}{l}\text { a. REPORT } \\
\text { Unclassified }\end{array}$ & $\begin{array}{l}\text { b. ABSTRACT } \\
\text { Unclassified }\end{array}$ & $\begin{array}{l}\text { c. THIS PAGE } \\
\text { Unclassified }\end{array}$ & & \\
\hline
\end{tabular}

19a. NAME OF RESPONSIBLE PERSON
19b. TELEPHONE NUMBER (Include area code)

